\title{
Maintaining Gauge Symmetry in Renormalizing Chiral Gauge Theories
}

\author{
Er-Cheng Tsa:* \\ Physics Department, National Taiwan University, Taipei, Taiwan
}

\begin{abstract}
It is known that the $\gamma_{5}$ scheme of Breitenlohner and Maison (BM) in dimensional regularization requires finite counter-term renormalization to restore gauge symmetry and implementing this finite renormalization in practical calculation is a daunting task even at 1-loop order. In this paper, we show that there is a simple and straightforward method to obtain these finite counter terms by using the rightmost $\gamma_{5}$ scheme in which we move all the $\gamma_{5}$ matrices to the rightmost position before analytically continuing the dimension. For any 1-loop Feynman diagram, the difference between the amplitude regularized in the rightmost $\gamma_{5}$ scheme and the amplitude regularized in the BM scheme can be easily calculated. The differences for all 1-loop diagrams in the chiral AbelianHiggs gauge theory and in the chiral non-Abelian gauge theory are shown to be the same as the amplitudes due to the finite counter terms that are required to restore gauge symmetry.
\end{abstract}

PACS numbers: 11.10.Gh, 11.15.Bt, 11.30.Rd

Keywords: $\gamma_{5}$; dimensional regularization; chiral fermion; renormalization

\footnotetext{
* ectsai@ntu.edu.tw
} 


\section{INTRODUCTION}

It is generally accepted that the original $\gamma_{5}$ dimensional regularization scheme proposed by 't Hooft and Veltman [1] and later systematized by Breitenlohner and Maison [2] can be used to regulate and renormalize chiral gauge theories in a rigorous manner. In this BM scheme, $\gamma_{5}$ is maintained as

$$
\gamma_{5}=i \gamma^{0} \gamma^{1} \gamma^{2} \gamma^{3}
$$

even when the space-time dimension $n$ departs from 4. Such $\gamma_{5}$ anti-commutes with $\gamma^{\mu}$ for $\mu$ in the first 4 dimensions but commutes with $\gamma^{\mu}$ when the index $\mu$ falls beyond the first 4 dimensions. As a consequence, an identity such as $\gamma_{5} \gamma^{\mu}=-\gamma^{\mu} \gamma_{5}$ in $n=4$ dimensional space no longer holds under dimensional regularization when the polarization $\mu$ is continued beyond the first 4 dimensions. The continuation to $n \neq 4$ for the Lagrangian of a theory with a gauge invariant 4 dimensional Lagrangian therefore depends on how we express and continue the terms involving product of $\gamma_{5}$ and $\gamma^{\mu}$ matrices in the Lagrangian. Furthermore, terms that are not gauge invariant in the $n$ dimensional Lagrangian must vanish when $n \rightarrow 4$ and thus contain a factor of $(n-4)$ or a $\gamma^{\mu}$ matrix with $\mu$ in the extra- 4 dimensions. Such gauge variant evanescent terms will contribute to the violation of gauge symmetry in the perturbative calculation of the theory under dimensional regularization.

The breakdown of gauge symmetry in the BM scheme can be remedied by introducing gauge variant local counter terms to restore the renormalized Ward identities [3] or BRST [4] gauge symmetry [5-9]. This procedure of removing spurious anomalies is usually a complicated and tedious task even at the 1-loop order. C. P. Martin and D. Sanchez-Ruiz [8] managed to successfully calculated the 1-loop finite counter terms needed for restoring gauge symmetry of the chiral non-Abelian gauge theory. For the chiral Abelian-Higgs theory, the 1-loop finite counter terms were later obtained by D. Sanchez-Ruiz [9] in which the laborious calculations were handled by computer routines.

In this paper, we shall present a simple and straightforward method for obtaining these finite counter terms. This is done with the help of the rightmost $\gamma_{5}$ scheme [10] in which the dimension $n$ is analytically continued after all the $\gamma_{5}$ matrices have been moved to the rightmost position. 


\section{THE RIGHTMOST $\gamma_{5}$ SCHEME}

For the QED theory, the identity

$$
\frac{1}{\ell+\not k-m} \not k \frac{1}{\ell-m}=\frac{1}{\ell-m}-\frac{1}{\ell+\not k-m}
$$

is the foundation that a Ward identity is built upon. For a gauge theory involving $\gamma_{5}$, there is a basic identity similar to (2) for verifying Ward identities:

$$
\frac{1}{\ell+\not k-m}(\not k-2 m) \gamma_{5} \frac{1}{\ell-m}=\gamma_{5} \frac{1}{\ell-m}+\frac{1}{\ell+\not k-m} \gamma_{5}
$$

The above identity valid at $n=4$ is derived by decomposing the vertex factor $(\not k-2 m) \gamma_{5}$ into $(\ell+\not k-m) \gamma_{5}$ and $\gamma_{5}(\ell-m)$ to annihilate respectively the propagators of the outgoing fermion with momentum $\ell+k$ and the incoming fermion with momentum $\ell$. Positioning $\gamma_{5}$ at the rightmost site, (3) becomes

$$
\frac{1}{\ell+\not k-m}(\not k-2 m) \frac{1}{-\ell-m} \gamma_{5}=\left(\frac{1}{-\ell-m}+\frac{1}{\ell+\not k-m}\right) \gamma_{5}
$$

If we disregard the rightmost $\gamma_{5}$ on both sides of the above identity, we obtain another identity

$$
\frac{1}{\ell+\not k-m}(\not k-2 m) \frac{1}{-\ell-m}=\frac{1}{-\ell-m}+\frac{1}{\ell+\not k-m}
$$

that is valid at $n=4$. This new identity (5), which is void of $\gamma_{5}$, may be analytically continued to hold when $n \neq 4$. We then multiply $\gamma_{5}$ on the right to every analytically continued term of this $\gamma_{5}$-free identity (15) to yield the analytic continuation of the identity (3).

As a side remark, we note that when we go to the dimension of $n \neq 4$, (3) in the form presented above is not valid. This is because $\gamma_{5}$ does not always anti-commute with $\gamma^{\mu}$ if $n \neq 4$. Adopting the rightmost $\gamma_{5}$ ordering avoids this difficulty, as the validity of the identity in the form of rightmost $\gamma_{5}$ ordering no longer depends on $\gamma_{5}$ anti-commuting with the $\gamma$ matrices.

Before analytic continuation is made, a $\gamma_{5}$-odd $\left(\gamma_{5}\right.$-even $)$ matrix product may always be reduced to a matrix product with only one (zero) $\gamma_{5}$ factor. For an amplitude corresponding to a diagram involving no fermion loops, we shall move all $\gamma_{5}$ matrices to the rightmost position before we continue analytically the dimension $n$. Subsequent application of dimensional regularization gives us regulated amplitudes satisfying the Ward identities. An 
identity relating the traces of matrix products without $\gamma_{5}$ at $n=4$ can always be analytically continued to hold when $n \neq 4$. Therefore, the portion of an amplitude in which the count of $\gamma_{5}$ on every fermion loop is even has no $\gamma_{5}$ difficulty and does not violate any Ward identity in this continuation scheme.

For any 1-loop Feynman diagram, the amplitude calculated according to the rightmost $\gamma_{5}$ scheme can be easily compared to that calculated according to the BM scheme. In fact, the difference between these two amplitudes can be straightforwardly calculated. If the rightmost $\gamma_{5}$ scheme is a gauge invariant scheme, we should be able to attribute the difference to the amplitude due to local counter terms that are required to restore BRST symmetry. It will be verified below with detailed results that this is indeed what happens. For the chiral Abelian-Higgs theory, the finite counter terms obtained by calculating the difference between the rightmost $\gamma_{5}$ scheme and the BM scheme are found to be exactly the same as those obtained in [9]. For the chiral non-Abelian gauge theory, the finite counter terms obtained in [8] can also be accounted for by the difference as demonstrated in Sec. VII. These results serve to confirm that the rightmost $\gamma_{5}$ scheme is indeed a gauge invariant regularization scheme.

\section{LAGRANGIAN FOR THE CHIRAL ABELIAN-HIGGS THEORY}

The BRST [4] invariant Lagrangian density for the chiral Abelian-Higgs gauge theory is

$$
\begin{aligned}
L_{B} & =-\frac{1}{4} F_{\mu \nu} F^{\mu \nu}+\left(D^{\mu} \phi\right)^{\dagger}\left(D_{\mu} \phi\right)-\frac{1}{2} \lambda g^{2}\left(\phi^{\dagger} \phi-\frac{1}{2} v^{2}\right)^{2} \\
& +\bar{\psi}_{L}(i \not D) \psi_{L}+\bar{\psi}_{R}(i \not \partial) \psi_{R}-\sqrt{2} f\left(\bar{\psi}_{L} \phi \psi_{R}+\bar{\psi}_{R} \phi^{\dagger} \psi_{L}\right) \\
& -\frac{1}{2 \alpha}\left(\partial_{\mu} A^{\mu}-\alpha \Lambda \phi_{2}\right)^{2}+i \bar{c}\left(\partial_{\mu} \partial^{\mu}+\alpha \Lambda M\right) c+i g \alpha \Lambda \bar{c} H c
\end{aligned}
$$

where $c$ is the ghost field, $\bar{c}$ is the anti-ghost field, and

$$
\begin{gathered}
F_{\mu \nu} \equiv \partial_{\mu} A_{\nu}-\partial_{\nu} A_{\mu}, \\
D_{\mu} \phi \equiv\left(\partial_{\mu}+i g A_{\mu}\right) \phi, \\
\psi_{L}=L \psi, \psi_{R}=R \psi
\end{gathered}
$$

with the chiral projection operators $L$ and $R$ defined as

$$
L=\frac{1}{2}\left(1-\gamma_{5}\right), R=\frac{1}{2}\left(1+\gamma_{5}\right) .
$$


The complex scalar field $\phi$ is related to real $H$ and $\phi_{2}$ by

$$
\phi=\frac{H+i \phi_{2}+v}{\sqrt{2}} .
$$

The Lagrangian $L_{B}$ of (6] $)$ is invariant under the BRST variations:

$$
\begin{aligned}
\delta_{B} A_{\mu} & =\partial_{\mu} c \\
\delta_{B} \phi_{2} & =-M c-g c H, \\
\delta_{B} H & =g c \phi_{2}, \\
\delta_{B} \psi_{L} & =-i g c \psi_{L}, \delta_{B} \psi_{R}=0, \\
\delta_{B} \bar{c} & =-\frac{i}{\alpha}\left(\partial^{\mu} A_{\mu}-\alpha \Lambda \phi_{2}\right), \delta_{B} c=0 .
\end{aligned}
$$

Define two mass parameters $M$ and $m$ by

$$
M=g v, m=f v
$$

Both $M$ and $m$ will be regarded as zero order quantities in perturbation.

Let us introduce the notation $\underline{p^{\mu}}$ for the component of $p^{\mu}$ vector in the first 4 dimensions and the notation $p_{\Delta}^{\mu}$ for the component in the remaining dimensions. i.e.,

$$
p^{\mu}=\underline{p}^{\mu}+p_{\Delta}^{\mu}
$$

with

$$
p_{\Delta}^{\mu}=0 \text { if } \mu \in\{0,1,2,3\}, \underline{p}^{\mu}=0 \text { if } \mu \notin\{0,1,2,3\} .
$$

Likewise, the Dirac matrix $\gamma^{\mu}$ is decomposed as

$$
\gamma^{\mu}=\underline{\gamma}^{\mu}+\gamma_{\Delta}^{\mu}
$$

with $\gamma_{\Delta}^{\mu}=0$ when $\mu \in\{0,1,2,3\}$ and $\underline{\gamma}^{\mu}=0$ when $\mu \notin\{0,1,2,3\}$. Since the definition (1) for $\gamma_{5}$ is valid even when the space-time dimension $n$ departs from 4 , we have

$$
\gamma_{5} \gamma^{\mu}+\gamma^{\mu} \gamma_{5}=2 \gamma_{\Delta}^{\mu} \gamma_{5}
$$

The free term involving the fermion fields in (6) is equal to

$$
\begin{aligned}
& \bar{\psi}_{L} i \not \partial \psi_{L}+\bar{\psi}_{R} i \not \partial \psi_{R}-f v\left(\bar{\psi}_{L} \psi_{R}+\bar{\psi}_{R} \psi_{L}\right) \\
& =\bar{\psi}(i R \not \partial L+i L \not \partial R-m) \psi \\
& =\bar{\psi}(i \not \partial-m) \psi
\end{aligned}
$$


where

$$
\underline{\not D}=\partial_{\mu} \underline{\gamma}^{\mu}=\partial_{\mu} \gamma^{\mu}-\partial_{\mu} \gamma_{\Delta}^{\mu}=\not \partial-\not \partial \Delta
$$

The fermion propagator corresponding to the free Lagrangian (11) is

$$
\frac{i}{\not p-m}
$$

which is independent of $p_{\Delta}$, the component of the momentum $p$ in the extra-4 dimensions and cannot be used for perturbative dimensional calculation.

To remedy this ill behavior, let us add the term

$$
E_{0}=\bar{\psi} i \not \partial_{\Delta} \psi=\bar{\psi}_{R} i \not \partial \psi_{L}+\bar{\psi}_{L} i \not \partial \psi_{R}
$$

to the BRST invariant $L_{B}$ of (6). The theory defined by the Lagrangian

$$
L_{e f f}=L_{B}+E_{0}
$$

will have well-behaved free fermion propagator

$$
\frac{i}{\not p-m}
$$

and can be used to calculate amplitudes perturbatively under the BM dimensional regularization scheme. By doing so, we also incur a loss of the BRST symmetry since $\delta_{B} L_{e f f}=$ $\delta_{B} E_{0} \neq 0$. Because $E_{0}$ vanishes as $n \rightarrow 4, E_{0}$ does not have any tree-level contribution. At one or more loop orders, simple $\frac{1}{n-4}$ pole factors or higher pole terms may arise from divergent loop integrals so that the contribution of $E_{0}$ cannot be neglected and additional local counter terms are required to restore the BRST symmetry.

For the Abelian theory with the Lagrangian (13), the propagators can be readily read off the free Lagrangian and vertex factors can be determined from the interaction terms in the Lagrangian. The propagators and vertices that are relevant to 1-loop finite counter-term calculation are listed in Appendix A 1.

\section{DIFFERENCE BETWEEN THE RIGHTMOST $\gamma_{5}$ SCHEME AND THE BM SCHEME IN THE ABELIAN-HIGGS THEORY.}

To illustrate how the counter-term amplitude is evaluated by calculating the difference between the rightmost $\gamma_{5}$ scheme and the BM scheme for the chiral Abelian-Higgs theory, 
consider the fermion self-energy diagram:

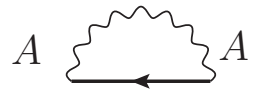

The horizontal line signifies an internal fermion line and the wavy line is a vector meson line. The Feynman amplitude in the BM scheme is

$$
\begin{aligned}
\Gamma^{B M} & =(-i g)^{2} \int \frac{d^{n} \ell}{(2 \pi)^{n}} D\left(A^{\mu}, A^{\nu} ; \ell\right) R \gamma^{\mu} L \frac{i}{\ell+\not p-m} R \gamma^{\nu} L \\
& =-i g^{2} \int \frac{d^{n} \ell}{(2 \pi)^{n}} D\left(A^{\mu}, A^{\nu} ; \ell\right) \frac{R \gamma^{\mu} L(\ell+\not p) R \gamma^{\nu} L}{(\ell+p)^{2}-m^{2}}
\end{aligned}
$$

where $\ell$ is the momentum of the internal vector meson line and the external momentum $p$ flowing into the fermion self-energy correction has only components in the first 4 dimensions. Anti-commuting $\gamma_{5}$ to the rightmost position, we obtain the corresponding amplitude in the rightmost $\gamma_{5}$ scheme:

$$
\Gamma^{R 5}=-i g^{2} \int \frac{d^{n} \ell}{(2 \pi)^{n}} D\left(A^{\mu}, A^{\nu} ; \ell\right) \frac{\gamma^{\mu}(\ell+\not p) \gamma^{\nu} L}{(\ell+p)^{2}-m^{2}}
$$

Both (15) and (16) are linearly divergent. Since the difference $\left(\Gamma^{R 5}-\Gamma^{B M}\right)$ contains at least a factor of $\gamma_{\Delta}$ matrix in the extra-4 dimensions, terms that are convergent will not survive the $n \rightarrow 4$ limit. We are free to change the mass pole of any propagator in evaluating $\left(\Gamma^{R 5}-\Gamma^{B M}\right)$ because the terms neglected are proportional to the mass square difference and are therefore convergent by power counting. Furthermore, if we expand the amplitude in a Taylor series with respect the external momentum $p$, terms proportional to $p^{N}$ with $N \geq 2$ are convergent and can be discarded in the difference between (15) and (16). i.e., we may substitute $-i \frac{\left(g^{\mu \nu}+(\alpha-1) \frac{\ell^{\mu} \ell^{\nu}}{\ell^{2}-m^{2}}\right)}{\left(\ell^{2}-m^{2}\right)^{2}}$ for $D\left(A^{\mu}, A^{\nu} ; \ell\right)$ and $\frac{1}{\ell^{2}-m^{2}}\left(1-\frac{2 \ell \cdot p}{\ell^{2}-m^{2}}\right)$ for $\frac{1}{(\ell+p)^{2}-m^{2}}$. The difference $\left(\Gamma^{R 5}-\Gamma^{B M}\right)$ after utilizing $R \gamma^{\mu} L=\underline{\gamma}^{\mu} L, R \gamma^{\nu} L=\underline{\gamma}^{\nu} L$ and $L \ell R=\underline{\ell R}$ can be written as

$$
\begin{aligned}
\lim _{n \rightarrow 4}\left(\Gamma^{R 5}-\Gamma^{B M}\right) & =-g^{2} \lim _{n \rightarrow 4} \int \frac{d^{n} \ell}{(2 \pi)^{n}} \frac{\left(g^{\mu \nu}+(\alpha-1) \frac{\ell^{\mu} \ell^{\nu}}{\ell^{2}-m^{2}}\right)\left(1-\frac{2 \ell \cdot p}{\ell^{2}-m^{2}}\right)}{\left(\ell^{2}-m^{2}\right)^{2}} \\
& \times\left(\gamma^{\mu}(\ell+\not p) \gamma^{\nu}-\underline{\gamma}^{\mu}(\underline{\ell}+\not p) \underline{\gamma}^{\nu}\right) L
\end{aligned}
$$

The symmetric integrals

$$
\begin{aligned}
\int d^{n} \ell f\left(\ell^{2}\right) \ell^{\mu} \ell^{\nu} & =\frac{g^{\mu \nu}}{n} \int d^{n} \ell f\left(\ell^{2}\right) \ell^{2}, \\
\int d^{n} \ell f\left(\ell^{2}\right) \ell^{\mu} \ell^{\nu} \ell^{\rho} \ell^{\sigma} & =\frac{\left(g^{\mu \nu} g^{\rho \sigma}+g^{\mu \rho} g^{\nu \sigma}+g^{\mu \sigma} g^{\rho \nu}\right)}{n(n+2)} \int d^{n} \ell f\left(\ell^{2}\right) \ell^{4}
\end{aligned}
$$


enable us to set

$$
\begin{aligned}
\int d^{n} \ell f\left(\ell^{2}\right)(\ell \cdot p) \ell & =\frac{1}{n} \int d^{n} \ell f\left(\ell^{2}\right) \ell^{2} \not p \\
\int d^{n} \ell f\left(\ell^{2}\right) \ell^{\mu} \ell^{\nu}(\ell \cdot p) \ell & =\frac{g^{\mu \nu} \not p+p^{\mu} \gamma^{\nu}+p^{\nu} \gamma^{\mu}}{n(n+2)} \int d^{n} \ell f\left(\ell^{2}\right) \ell^{4}
\end{aligned}
$$

and reduce (17) to

$$
\begin{aligned}
\lim _{n \rightarrow 4}\left(\Gamma^{R 5}-\Gamma^{B M}\right) & =\frac{g^{2}}{6} \lim _{n \rightarrow 4} \int \frac{d^{n} \ell}{(2 \pi)^{n}} \frac{(n-4)}{\left(\ell^{2}-m^{2}\right)^{2}}(1+2 \alpha) \not p L \\
& =-\frac{1}{(4 \pi)^{2}} i \frac{g^{2}}{3}(1+2 \alpha) \not p L
\end{aligned}
$$

where we have also utilized the integral

$$
\lim _{n \rightarrow 4} \int \frac{d^{n} \ell}{(2 \pi)^{n}} \frac{(n-4)}{\left(\ell^{2}-m\right)^{2}}=4 \int \frac{d^{4} \ell}{(2 \pi)^{4}} \frac{m^{2}}{\left(\ell^{2}-1\right)^{3}}=\frac{-2 i}{(4 \pi)^{2}}
$$

In the BM scheme, this amplitude (18) can be accounted for by adding the counter term

$$
-\frac{1}{(4 \pi)^{2}} \frac{g^{2}}{3}(1+2 \alpha) \bar{\psi} R i \not \partial L \psi
$$

to the Lagrangian (13).

The counter-term amplitude for any divergent 1-loop 1PI diagram can be similarly calculated. The diagrams that are responsible for all 1-loop counter terms are listed in Figure 1- 7 in Appendix $\mathrm{A}$. The corresponding counter-term amplitudes are calculated and summarized in Table IV- $\mathrm{X}$.

\section{1-LOOP RESULTS FOR THE CHIRAL ABELIAN-HIGGS THEORY}

For the chiral Abelian-Higgs theory, D. Sanchez-Ruiz [9] has successfully computed in the BM scheme the 1-loop finite counter terms that are required to restore the BRST symmetry by evaluating the terms that break the Slavnov-Taylor identities and then solving a linear system of 27 equations with 32 variables to find a solution. The calculation in [9] is rather cumbersome and has to be relied on computer routines. The general solution for the 1-loop finite counter terms is given by (30) of [9]

$$
\hbar \tilde{S}_{f c t}^{(1)}=-\sum_{i=1}^{32} \hbar \tilde{x}_{0, i}^{(1)} \tilde{e}_{i}+\hbar \sum_{l=1}^{11} c_{l}^{(1)} \mathcal{I}_{l}
$$


where each $\mathcal{I}_{l}, i=1,2 . ., 11$ is BRST invariant and $\tilde{e}_{i}, i=1,2 . ., 32$ given by (16) in [9] form a basis of the space of the integrated Lorentz scalar CP-invariant polynomials in the fields and their derivatives with maximal canonical dimension 4 and ghost number 0 . A particular solution for the coefficients $\tilde{x}_{0, i}^{(1)}$ is given by (29) in [9] and tabulated in the 2nd column of Table I, II and III. Counter terms that involve fermion fields are listed in Table II. Otherwise, they are listed in Table 【I and 【II.

\begin{tabular}{|l|l|l|l|}
\hline$\tilde{e}_{i}$ & $-(4 \pi)^{2} \tilde{x}_{0, i}^{(1)}$ & $\begin{array}{l}\xi^{\prime}=\alpha, \rho=-\alpha \Lambda, \\
\theta=0, r=1\end{array}$ & $\begin{array}{l}\text { rightmost } \gamma_{5} \\
\text { method }\end{array}$ \\
\hline$\tilde{e}_{24}=\bar{\psi} \psi$ & $-\frac{f\left[3 \rho r+4 g^{2} \theta(1+\theta r) v\left(5+\xi^{\prime}\right)\right]}{6 r}$ & $\frac{1}{2} \alpha \Lambda f$ & $-\frac{1}{2} \alpha \Lambda f g$ \\
$\tilde{e}_{25}=\bar{\psi} i \not \partial L \psi$ & 0 & 0 & 0 \\
$\tilde{e}_{26}=\bar{\psi} i \not \partial R \psi$ & 0 & 0 & 0 \\
$\tilde{e}_{27}=\bar{\psi} / A L \psi$ & $-\frac{\left[-6 f^{2} r+g^{2}\left(2 \theta+r+\theta^{2} r\right)\left(5+\xi^{\prime}\right)\right]}{6}$ & $f^{2}-\frac{1}{6} g^{2}(5+\alpha)$ & $-f^{2} g+\frac{1}{6} g^{3}(5+\alpha)$ \\
$\tilde{e}_{28}=\bar{\psi} / A R \psi$ & $\frac{r\left[-6 f^{2}+g^{2} \theta^{2}\left(5+\xi^{\prime}\right)\right]}{6}$ & $-f^{2}$ & $f^{2} g$ \\
$\tilde{e}_{29}=\bar{\psi} H \psi$ & $-\frac{2}{3} f g^{2} \theta(\theta+r)\left(5+\xi^{\prime}\right)$ & 0 & 0 \\
$\tilde{e}_{30}=\bar{\psi} \phi_{2} \gamma_{5} \psi$ & 0 & 0 & 0 \\
\hline
\end{tabular}

TABLE I. Counter terms due to diagrams with open fermion lines

The theory defined by the $L_{\text {eff }}$ of (13) corresponds to the theory of (5) in [9] with $\xi^{\prime}=\alpha, \rho=-\alpha \Lambda, \theta=0$ and $r=1$. Correspondingly, the 3rd column of Table $\llbracket$ is obtained from the 2nd column with the substitution $\xi^{\prime}=\alpha, \rho=-\alpha \Lambda, \theta=0$ and $r=1$.

From Table IV, V, VI and VII, the sum of counter terms due to the diagrams from Figure 1. 2, 3, and 4 obtained according to the method of evaluating the difference between the rightmost $\gamma_{5}$ scheme and the $\mathrm{BM}$ scheme is equal to

$$
\frac{1}{(4 \pi)^{2}}\left[\begin{array}{c}
-\frac{g^{2}}{3}(1+2 \alpha) \bar{\psi} R i \not \partial L \psi+\frac{1}{2} \alpha(M-\Lambda) f g \bar{\psi} \psi \\
\frac{1}{6} g^{3}(7+5 \alpha) \bar{\psi} R / A L \psi+f^{2} g \bar{\psi} / A \gamma_{5} \psi+\frac{\alpha g^{2} f}{2} \bar{\psi}\left(H+i \phi_{2} \gamma_{5}\right) \psi
\end{array}\right]
$$

which after subtracting out the gauge invariant term

$$
\frac{1}{(4 \pi)^{2}}\left[-\frac{g^{2}}{3}(1+2 \alpha) \bar{\psi} R(i \not \partial-g / A) L \psi+\frac{\alpha g^{2} f}{2} \bar{\psi}\left(H+v+i \phi_{2} \gamma_{5}\right) \psi\right]
$$

becomes

$$
\frac{1}{(4 \pi)^{2}}\left[-\frac{1}{2} \alpha \Lambda f g \bar{\psi} \psi+\left(\frac{1}{6} g^{3}(5+\alpha)-f^{2} g\right) \bar{\psi} R / A L \psi+f^{2} g \bar{\psi} / A R \psi\right]
$$


The above expression (22) decomposed as a linear combination of $\tilde{e}_{i}$ is listed under the column "rightmost $\gamma_{5}$ method" of Table I. The Lagrangian (13) is defined differently from that in [9]. The covariant derivative $D_{\mu}$ is defined as $D_{\mu}=\partial_{\mu}+i g A_{\mu}$ for the theory (13) but as $D_{\mu}=\partial_{\mu}-i A_{\mu}$ in [9]. The vector field $A$ in a counter-term expression obtained in [9] needs to be scaled to $-g A$ to be identified as the corresponding counter term for the theory of (13). There is a single vector $A$ field in $\tilde{e}_{27}=\bar{\psi} / A L \psi$ or $\tilde{e}_{28}=\bar{\psi} / A R \psi$. As a consequence, multiplying $-g$ to the coefficient of either $\tilde{e}_{27}$ or $\tilde{e}_{28}$ under the 3rd column of Table \should give us the corresponding coefficient under the column "rightmost $\gamma_{5}$ method". Furthermore, the counter term proportional to $\tilde{e}_{24}=\bar{\psi} \psi$ actually stems from diagram $(d)$ and $(e)$ of Figure 1. The ratio of the coefficient of $\tilde{e}_{24}$ for the theory of (13) over that obtained in [9] as shown in Table I is equal to $-g$ and can be accounted for by the ratio of vertex factors due to the single $\bar{\psi}-A-\psi$ vertex in each of the diagram $(d)$ and $(e)$ of Figure 1 . Table \in fact shows that the 1-loop counter terms that involve fermion fields calculated by the rightmost $\gamma_{5}$ method are in agreement with those obtained in [9].

The 1-loop counter terms due to the diagrams without external fermion lines from Figure [5, 6] and 7 are summarized in Table VIII, IX] and X]. The total of these counter terms is

$$
\frac{1}{(4 \pi)^{2}}\left[\begin{array}{c}
4 m^{2} f^{2}\left(\phi_{2}\right)^{2}-\frac{4}{3} f^{2}\left(\partial \phi_{2}\right)^{2}-g^{2} m^{2} A^{2}+\frac{1}{6} g^{2}\left(\partial_{\mu} A_{\nu}\right)\left(\partial^{\mu} A^{\nu}\right) \\
+8 f^{3} m H\left(\phi_{2}\right)^{2}-2 f g^{2} m H A^{2}+4 f^{2} g \phi_{2}\left(\partial_{\mu} H\right) A^{\mu} \\
+4 f^{4} H^{2}\left(\phi_{2}\right)^{2}+\frac{8}{3} f^{4}\left(\phi_{2}\right)^{4}-f^{2} g^{2} H^{2} A^{2}-3 f^{2} g^{2}\left(\phi_{2}\right)^{2} A^{2}+\frac{1}{12} g^{4}\left(A^{2}\right)^{2}
\end{array}\right]
$$

and can be written as the sum of

$$
\frac{1}{(4 \pi)^{2}}\left[2 f^{4}\left((H+v)^{2}+\left(\phi_{2}\right)^{2}\right)^{2}-2 f^{4} v^{4}+\frac{1}{12} g^{2} F_{\mu \nu} F^{\mu \nu}\right]
$$

and

$$
\frac{1}{(4 \pi)^{2}}\left[\begin{array}{c}
-2 f^{4}\left(H^{4}+4 v H^{3}+6 v^{2} H^{2}+4 v^{3} H\right)+\frac{2}{3} f^{4}\left(\phi_{2}\right)^{4}-\frac{2}{3} f^{2}\left(\partial \phi_{2}\right)^{2} \\
+\frac{1}{6} g^{2}\left(\partial_{\mu} A^{\mu}\right)^{2}-g^{2} m^{2} A^{2}-2 f g^{2} m H A^{2}+4 f^{2} g \phi_{2}\left(\partial_{\mu} H\right) A^{\mu} \\
-f^{2} g^{2} H^{2} A^{2}+\frac{1}{12} g^{4}\left(A^{2}\right)^{2}-3 f^{2} g^{2}\left(\phi_{2}\right)^{2} A^{2}
\end{array}\right] .
$$

(24) is gauge invariant and (25) expressed as a linear combination of $\tilde{e}_{i}$ is tabulated in Table II and III under the column "rightmost $\gamma_{5}$ method" while the result from [9] is listed under the column $-(4 \pi)^{2} \tilde{x}_{0, i}^{(1)}$. Taking into the consideration of the $-g$ factor for each vector field $A$ in comparing the counter-term expressions, the counter terms listed in Table II and III obtained by the rightmost $\gamma_{5}$ method for the theory of (13) are in exact agreement with those obtained in [9]. 


\begin{tabular}{|l|l|l|}
\hline$\tilde{e}_{i}$ & $-(4 \pi)^{2} \tilde{x}_{0, i}^{(1)}$ & rightmost $\gamma_{5}$ method \\
\hline$\tilde{e}_{1}=H$ & $-8 f^{4} v^{3}$ & $-8 f^{4} v^{3}$ \\
$\tilde{e}_{2}=H^{2}$ & $-12 f^{4} v^{2}$ & $-12 f^{4} v^{2}$ \\
$\tilde{e}_{3}=\left(\phi_{2}\right)^{2}$ & 0 & 0 \\
$\tilde{e}_{4}=H^{3}$ & $-8 f^{4} v$ & $-8 f^{4} v$ \\
$\tilde{e}_{5}=H\left(\phi_{2}\right)^{2}$ & 0 & 0 \\
$\tilde{e}_{6}=H^{4}$ & $-2 f^{4}$ & $-2 f^{4}$ \\
$\tilde{e}_{7}=\left(\phi_{2}\right)^{4}$ & 0 & $\frac{2}{3} f^{4}$ \\
$\tilde{e}_{8}=H^{2}\left(\phi_{2}\right)^{2}$ & 0 \\
$\tilde{e}_{9}=\left(\partial_{\mu} H\right)\left(\partial^{\mu} H\right)$ & 0 & 0 \\
$\tilde{e}_{10}=\left(\partial_{\mu} \phi_{2}\right)\left(\partial^{\mu} \phi_{2}\right)$ & $-\frac{2}{3} f^{2}$ & $-\frac{2}{3} f^{2}$ \\
\hline
\end{tabular}

TABLE II. Counter terms without A fields due to diagrams with a closed fermion loop

\begin{tabular}{|l|l|l|}
\hline$\tilde{e}_{i}$ & $-(4 \pi)^{2} \tilde{x}_{0, i}^{(1)}$ & rightmost $\gamma_{5}$ method \\
\hline$\tilde{e}_{11}=\phi_{2}\left(\partial_{\mu} A^{\mu}\right)$ & 0 & 0 \\
$\tilde{e}_{12}=A_{\mu} H\left(\partial^{\mu} \phi_{2}\right)$ & 0 & 0 \\
$\tilde{e}_{13}=A_{\mu} \phi_{2}\left(\partial^{\mu} H\right)$ & $-4 f^{2}$ & $4 f^{2} g$ \\
$\tilde{e}_{14}=A_{\mu} A^{\mu}$ & $-f^{2} v^{2}$ & $-f^{2} g^{2} v^{2}$ \\
$\tilde{e}_{15}=A_{\mu} A^{\mu} H$ & $-2 f^{2} v$ & $-2 f^{2} g^{2} v$ \\
$\tilde{e}_{16}=A_{\mu} A^{\mu} H^{2}$ & $-f^{2}$ & $-f^{2} g^{2}$ \\
$\tilde{e}_{17}=A_{\mu} A^{\mu}\left(\phi_{2}\right)^{2}$ & $-3 f^{2}$ & $-3 f^{2} g^{2}$ \\
$\tilde{e}_{18}=\left(\partial_{\mu} A^{\mu}\right)^{2}$ & $\frac{1}{6}$ & $\frac{1}{6} g^{2}$ \\
$\tilde{e}_{19}=F_{\mu \nu} F^{\mu \nu}$ & 0 & 0 \\
$\tilde{e}_{20}=\left(A_{\mu} A^{\mu}\right)^{2}$ & $\frac{1}{12}$ & $\frac{1}{12} g^{4}$ \\
\hline
\end{tabular}

TABLE III. Counter terms involving A fields due to diagrams with a closed fermion loop 


\section{LAGRANGIAN FOR THE CHIRAL NON-ABELIAN GAUGE THEORY}

The BRST invariant Lagrangian density for the chiral non-Abelian gauge theory is

$$
\begin{aligned}
\tilde{L}_{B} & =-\frac{1}{4} F_{\mu \nu}^{a} F^{a, \mu \nu}+\bar{\psi}_{L} i \not D \psi_{L}+\bar{\psi}_{R} i \not \partial \psi_{R}+i \bar{\psi}_{R}^{\prime} i \not D \psi_{R}^{\prime}+\bar{\psi}_{L}^{\prime} i \not \partial \psi_{L}^{\prime} \\
& -\frac{1}{2 \alpha}\left(\partial^{\mu} A_{\mu}^{a}\right)\left(\partial^{\nu} A_{\nu}^{a}\right)+i \bar{c}^{a} \square c^{a}+i g C^{a b c}\left(\partial^{\mu} \bar{c}^{a}\right) A_{\mu}^{b} c^{c}
\end{aligned}
$$

where $c^{a}$ is the ghost field, $\bar{c}^{a}$ is the anti-ghost field, and

$$
F_{\mu \nu}^{a}=\partial_{\mu} A_{\nu}^{a}-\partial_{\nu} A_{\mu}^{a}-g C^{a b c} A_{\mu}^{b} A_{\nu}^{c}
$$

Two fermion fields $\psi$ and $\psi^{\prime}$ whose left-handed component $\psi_{L}=L \psi$ and right-handed component $\psi_{R}^{\prime}=R \psi^{\prime}$ are coupled to $A_{\mu}^{a}$. The covariant derivatives for $\psi_{L}$ and $\psi_{R}^{\prime}$ are

$$
\begin{aligned}
D_{\mu} \psi_{L} & =\left(\partial_{\mu}+i g A_{\mu}^{a} T_{L}^{a}\right) \psi_{L} \\
D_{\mu} \psi_{R}^{\prime} & =\left(\partial_{\mu}+i g A_{\mu}^{a} T_{R}^{a}\right) \psi_{R}^{\prime}
\end{aligned}
$$

where $T_{L}^{a}\left(T_{R}^{a}\right)$ are group generators that satisfy

$$
\begin{aligned}
{\left[T_{L}^{a}, T_{L}^{b}\right] } & =i C^{a b c} T_{L}^{c},\left[T_{R}^{a}, T_{R}^{b}\right]=i C^{a b c} T_{R}^{c} \\
\operatorname{tr}\left(T_{L}^{a} T_{L}^{b}\right) & =T_{L} \delta^{a b}, \operatorname{tr}\left(T_{R}^{a} T_{R}^{b}\right)=T_{R} \delta^{a b} \\
\sum_{e} T_{L}^{e} T_{L}^{e} & =C_{L}, \sum_{e} T_{R}^{e} T_{R}^{e}=C_{R}
\end{aligned}
$$

For convenience, we also adopt the following shorthand notations defined in [8]:

$$
\operatorname{tr}\left(T_{L}^{a} T_{L}^{b} T_{L}^{c} T_{L}^{d}\right)=T_{L}^{a b c d}, \operatorname{tr}\left(T_{R}^{a} T_{R}^{b} T_{R}^{c} T_{R}^{d}\right)=T_{R}^{a b c d}
$$

The Lagrangian (26) is invariant under the BRST variations:

$$
\begin{aligned}
\delta_{B} A_{\mu}^{a} & =\partial_{\mu} c^{a}+g C^{a b c} c^{b} A_{\mu}^{c} \\
\delta_{B} \psi_{L} & =-i c^{a} T_{L}^{a} \psi_{L}, \delta_{B} \psi_{R}=0 \\
\delta_{B} \psi_{R}^{\prime} & =-i c^{a} T_{R}^{a} \psi_{R}^{\prime}, \delta_{B} \psi_{L}^{\prime}=0 \\
\delta_{B} c^{a} & =\frac{1}{2} g C^{a b c} c^{b} c^{c} \\
\delta_{B} \bar{c}^{a} & =\frac{1}{i \alpha} \partial_{\mu} A^{a, \mu}
\end{aligned}
$$

As with the chiral Abelian-Higgs theory (13), a gauge variant evanescent term

$$
\tilde{E}_{0}=\bar{\psi} i \not \partial_{\Delta} \psi+\bar{\psi}^{\prime} i \not \partial_{\Delta} \psi^{\prime}
$$


needs to be added the BRST invariant (26) to define the Lagrangian

$$
\tilde{L}_{e f f}=\tilde{L}_{B}+\tilde{E}_{0}
$$

for the chiral non-Abelian gauge theory that can be calculated perturbatively in the BM

scheme. For the non-Abelian theory (28), the propagators and vertices that are relevant to 1-loop finite counter-term calculation are listed in Appendix B 1.

\section{1-LOOP RESULTS FOR THE CHIRAL NON-ABELIAN GAUGE THEORY}

C. P. Martin and D. Sanchez-Ruiz have obtained with tedious calculations the 1-loop finite counter terms that are needed for restoring BRST symmetry in the BM dimensional regularization formalism for the chiral non-Abelian gauge theory with the result given in (69) of [8]. In Appendix [B, the 1-loop counter terms for this non-Abelian theory are computed straightforwardly by evaluating the difference of amplitudes between the rightmost $\gamma_{5}$ scheme and the BM scheme with the results summarized in Table XI. Specifically, diagrams in Figure 8 and 9 yield the counter terms that involve fermion fields and can be written as

$$
\frac{1}{(4 \pi)^{2}}\left[\begin{array}{c}
-\frac{1}{3} g^{2}(1+2 \alpha)\left(\bar{\psi}_{L} i \not \partial \psi_{L} C_{L}+\bar{\psi}_{R}^{\prime} i \not \partial \psi_{R}^{\prime} C_{R}\right) \\
+\frac{1}{6} g^{3}(7+5 \alpha)\left(\bar{\psi}_{L} / A \psi_{L} C_{L} T_{L}^{a}+\bar{\psi}_{R}^{\prime} / A \psi_{R}^{\prime} C_{R} T_{R}^{a}\right)
\end{array}\right]
$$

Subtracting out the gauge invariant term

$$
\frac{1}{(4 \pi)^{2}}\left[-i \frac{1}{6} g^{2}(7+5 \alpha)\left(\begin{array}{c}
\bar{\psi}_{L}\left(\not \partial+i g / A^{a} T_{L}^{a}\right) \psi_{L} C_{L} T_{L}^{a} \\
+\bar{\psi}_{R}^{\prime}\left(\not \partial+i g / A^{a} T_{R}^{a}\right) \psi_{R}^{\prime} C_{R} T_{R}^{a}
\end{array}\right)\right]
$$

from (29), we get

$$
\frac{1}{(4 \pi)^{2}}\left[\left(1+\frac{(\alpha-1)}{6}\right) g^{2}\left(\bar{\psi}_{L} i \not \partial \psi_{L} C_{L} T_{L}^{a}+\bar{\psi}_{R}^{\prime} i \not \partial \psi_{R}^{\prime} C_{R} T_{R}^{a}\right)\right]
$$

which, after the identification of $\alpha$ with $\alpha^{\prime}$, is consistent with the finite counter-terms (69) of $[8]$.

Figure 10] 11, and 12 are responsible for the counter terms that are free of fermion fields. From Table XI, the sum of these counter terms is equal to

$$
\frac{1}{(4 \pi)^{2}}\left[\begin{array}{c}
-\frac{1}{6} g^{2}\left(T_{L}+T_{R}\right) A_{\mu}^{a} \square A^{a, \mu} \\
-\frac{2}{3} g^{3}\left(T_{L}+T_{R}\right) C^{a b c}\left(\partial^{\mu} A_{\nu}^{a}\right) A_{\mu}^{b} A^{c, \nu} \\
\frac{1}{12} g^{4}\left(T_{L}^{a b c d}+T_{R}^{a b c d}\right) A^{a, \mu} A_{\mu}^{b} A^{c, \nu} A_{\nu}^{d} \\
+\frac{5}{24} g^{4}\left(T_{L}+T_{R}\right) C^{e a b} C^{e c d} A_{\mu}^{a} A_{\nu}^{b} A^{c, \mu} A^{d, \nu}
\end{array}\right]
$$


which can be written as the sum of the gauge invariant term

$$
\frac{5}{24} g^{2}\left(T_{L}+T_{R}\right) F_{\mu \nu}^{a} F^{a, \mu \nu}
$$

and

$$
\frac{1}{(4 \pi)^{2}}\left[\begin{array}{c}
\left(T_{L}+T_{R}\right) g^{2}\left(\frac{5}{12}(\partial A)^{2}+\frac{1}{4} A \square A\right) \\
+\frac{\left(T_{L}+T_{R}\right)}{6} g^{3} C^{a b c}\left(\partial_{\mu} A_{\nu}^{a}\right) A_{\mu}^{b} A_{\nu}^{c} \\
+\frac{1}{12} g^{4}\left(T_{L}^{a b c d}+T_{R}^{a b c d}\right) A^{a, \mu} A_{\mu}^{b} A^{c, \nu} A_{\nu}^{d}
\end{array}\right]
$$

Upon the scaling of $A \rightarrow-g A$, the chiral non-Abelian gauge theory with the tree action defined by (32) in [8] becomes the non-Abelian theory defined by (26). Taking into the consideration of this $A \rightarrow-g A$ scaling, (32) is also in agreement with (69) of [8].

\section{CONCLUSION}

In the BM scheme, simply removing the pole terms from the amplitudes of 1-loop diagrams does not yield renormalized amplitudes that satisfy Ward identities. Instead, some finite renormalization terms have to be added. These finite counter terms are determined from restoring the validities of Ward identities. Implementing this finite renormalization in practical calculation is usually a daunting task even at 1-loop order.

For the chiral Abelian-Higgs gauge theory and the chiral non-Abelian Yang-Mills theory, we have verified that the renormalized amplitudes for all 1-loop diagrams calculated in the BM scheme with finite counter term renormalization are equal to those obtained directly in the rightmost $\gamma_{5}$ scheme. This means we can be spared the tedious finite renormalization procedures if the rightmost $\gamma_{5}$ scheme is adopted. Furthermore, since all the $\gamma_{5}$ matrices are moved to and consolidated at a single position before continuing the dimension in our scheme, the burden of evaluating the matrix products or trace of matrix products is considerably less than that in the BM scheme. In our opinion, this rightmost $\gamma_{5}$ prescription is a much simpler scheme than the BM scheme for calculating amplitudes in gauge theories involving $\gamma_{5}$.

For the rightmost $\gamma_{5}$ scheme, the prescription that leads to the preservation of Ward identities makes no use of the specific type of gauge theories in question. As a consequence, the rightmost $\gamma_{5}$ scheme should be applicable for any type of chiral gauge theory, in particular, the standard model. 


\section{Appendices}

\section{Appendix A: The Chiral Abelian-Higgs Theory}

\section{Feynman Rules}

The propagators and vertices used in the 1-loop counter-term calculation for the chiral Abelian-Higgs theory defined by (13) are listed below.

a. Propagators:

$$
\begin{aligned}
& S(\psi, \bar{\psi} ; p): \stackrel{p}{\longleftarrow}=\frac{i}{\not p-m} \\
& D\left(A^{\mu}, A^{\nu} ; k\right): \overbrace{\mu}^{\sim_{\nu}^{k}}=-i\left(\frac{g^{\mu \nu}-\frac{k^{\mu} k^{\nu}}{k^{2}}}{k^{2}-M^{2}}+\frac{\alpha\left(k^{2}-\alpha \Lambda^{2}\right) k^{\mu} k^{\nu}}{k^{2}\left(k^{2}-\alpha \Lambda M\right)^{2}}\right) \\
& D\left(A^{\mu}, \phi_{2} ; k\right): \sim_{\mu}^{\leftarrow \kappa}{ }_{\phi_{2}}^{\leftarrow}=\frac{\alpha(M-\Lambda) k^{\mu}}{\left(k^{2}-\alpha \Lambda M\right)^{2}}
\end{aligned}
$$

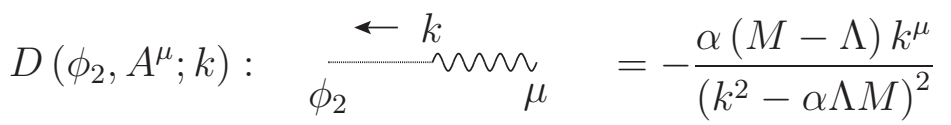

$$
\begin{aligned}
& D\left(\phi_{2}, \phi_{2} ; k\right): \underset{\phi_{2}}{\leftarrow k} \phi_{2}=\frac{i\left(k^{2}-\alpha M^{2}\right)}{\left(k^{2}-\alpha \Lambda M\right)^{2}} \\
& D(H, H ; k): \frac{\leftarrow k}{H} \quad \stackrel{\leftarrow}{H}=\frac{i}{k^{2}-\lambda M^{2}}
\end{aligned}
$$

b. Vertex Factors:

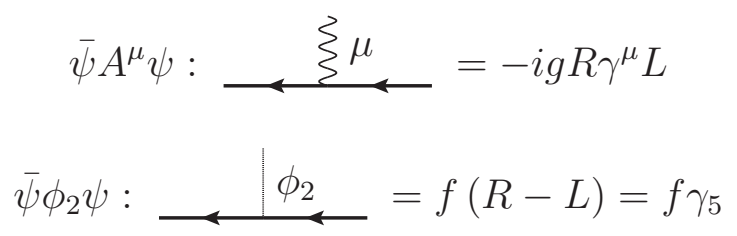




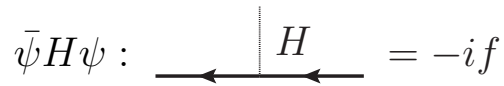

$$
\begin{aligned}
& H A^{\mu} \phi_{2}: \quad \sum_{H} \sum_{\phi_{2}}^{k} \mu \quad p\left(2 p^{\mu}+k^{\mu}\right)
\end{aligned}
$$

\section{1-Loop Counter Terms}

a. Figure [1: $\bar{\psi} \psi$ Self-Energy Diagrams

The possible diagrams that may contribute to the fermion self-energy are depicted in Figure 1.

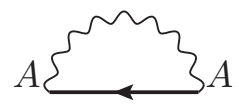

(a)

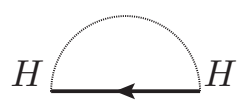

(b)

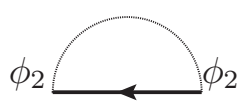

$(c)$

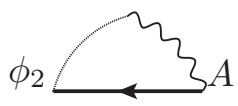

$(d)$

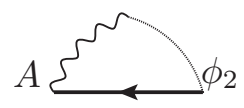

$(e)$

FIG. 1. Fermion Self-Energy Diagrams

In each diagram of Figure 1, the horizontal line signifies an internal fermion line and the wavy line is a vector meson line.

Diagram $(a)$

This diagram has been discussed thoroughly in Sec. IV] The Feynman amplitude in the BM scheme is denoted by $\Gamma_{\square}^{B M} a{ }^{B}$.

$$
\Gamma_{\mathbb{1}_{(a)}^{B M}}^{B M}=-i g^{2} \int \frac{d^{n} \ell}{(2 \pi)^{n}} D\left(A^{\mu}, A^{\nu} ; \ell\right) R \gamma^{\mu} L \frac{1}{\ell+\not p-m} R \gamma^{\nu} L
$$

The corresponding amplitude in the rightmost $\gamma_{5}$ scheme is denoted by $\Gamma_{\square[a}^{R 5}$.

$$
\Gamma_{\square_{(a)}^{R 5}}^{R 5}=-i g^{2} \int \frac{d^{n} \ell}{(2 \pi)^{n}} D\left(A^{\mu}, A^{\nu} ; \ell\right) \gamma^{\mu} \frac{\ell+\not p}{(\ell+p)^{2}-m^{2}} \gamma^{\nu} L
$$

The difference has been shown in Sec. IV to be

$$
\lim _{n \rightarrow 4}\left(\Gamma_{\mathbb{1}_{(a)}^{R 5}}-\Gamma_{\mathbb{1}_{(a)}^{B M}}^{B M}\right)=-\frac{1}{(4 \pi)^{2}} i \frac{g^{2}}{3}(1+2 \alpha) \not p L
$$


Diagram $(b)$

There is no $\gamma_{5}$ in the BM amplitude $\Gamma_{1_{b}(b)}^{B M}$. The rightmost $\gamma_{5}$ amplitude $\Gamma_{\left.\mathbb{1}_{b}\right)}^{R 5}$ is the same

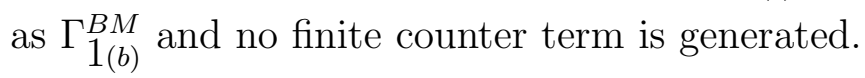

$$
\Gamma_{[1 b)}^{R 5}-\Gamma_{[1(b)}^{B M}=0
$$

Diagram $(c)$

$$
\begin{aligned}
& \Gamma_{\left[\mathbb{1}_{(c)}^{B M}\right.}^{B M}=f^{2} \int \frac{d^{n} \ell}{(2 \pi)^{n}} D\left(\phi_{2}, \phi_{2}, \ell\right) \gamma_{5} \frac{i}{\ell+\not p-m} \gamma_{5} \\
& \Gamma_{1_{(c)}^{R 5}}^{R}=f^{2} \int \frac{d^{n} \ell}{(2 \pi)^{n}} D\left(\phi_{2}, \phi_{2}, \ell\right) \frac{i}{-\ell-\not p-m} \\
& \lim _{n \rightarrow 4}\left(\Gamma_{[1](c)}^{R 5}-\Gamma_{[1](c)}^{B M}\right) \\
& =i f^{2} \int \frac{d^{n} \ell}{(2 \pi)^{n}} D\left(\phi_{2}, \phi_{2}, \ell\right)\left(\frac{-\not \ell-\not p+m-\gamma_{5}(\ell+\not p+m) \gamma_{5}}{(\ell+p)^{2}-m^{2}}\right) \\
& =i f^{2} \int \frac{d^{n} \ell}{(2 \pi)^{n}} D\left(\phi_{2}, \phi_{2}, \ell\right) \frac{-2 \ell_{\Delta}}{(\ell+p)^{2}-m^{2}}=0
\end{aligned}
$$

$\operatorname{Diagram}(d)$

$$
\begin{aligned}
& \Gamma_{\underline{1}_{d)}^{B M}}^{B M}=(-i g) f \int \frac{d^{n} \ell}{(2 \pi)^{n}} D\left(A^{\mu}, \phi_{2} ; \ell\right) \gamma_{5} \frac{i}{\ell+\not p-m} R \gamma^{\mu} L \\
& \Gamma_{1_{d)}^{R 5}}^{R 5}=(-i g) f \int \frac{d^{n} \ell}{(2 \pi)^{n}} D\left(A^{\mu}, \phi_{2} ; \ell\right) \frac{i}{-\ell-\not p-m} \gamma^{\mu} L \\
& \lim _{n \rightarrow 4}\left(\Gamma_{\Gamma_{(1)}^{R 5}}^{R l_{d)}}-\Gamma_{[]_{d)}^{B M}}^{B(d)}\right) \\
& =g f \lim _{n \rightarrow 4} \int \frac{d^{n} \ell}{(2 \pi)^{n}} D\left(A^{\mu}, \phi_{2} ; \ell\right) \frac{-\ell-\gamma_{5} \ell R}{\ell^{2}-m^{2}} \gamma^{\mu} L \\
& =\alpha(M-\Lambda) g f \lim _{n \rightarrow 4} \int \frac{d^{n} \ell}{(2 \pi)^{n}} \frac{-\ell-\gamma_{5} \ell R}{\left(\ell^{2}-m\right)^{3}} \not L \\
& =-\alpha(M-\Lambda) g f \lim _{n \rightarrow 4} \int \frac{d^{n} \ell}{(2 \pi)^{n}} \frac{\ell_{\Delta}^{2}}{\left(\ell^{2}-m\right)^{3}} L=\frac{1}{(4 \pi)^{2}} \frac{i}{2} \alpha(M-\Lambda) f g L
\end{aligned}
$$

Diagram (e)

$$
\begin{gathered}
\Gamma_{\mathbb{1}_{(e)}^{B M}}^{R}=(-i g) f \int \frac{d^{n} \ell}{(2 \pi)^{n}} D\left(\phi_{2}, A^{\mu} ; \ell\right) R \gamma^{\mu} L \frac{i}{\ell+\not p-m} \gamma_{5} \\
\Gamma_{\mathbb{1}_{(e)}^{R 5}}^{R}=g f \int \frac{d^{n} \ell}{(2 \pi)^{n}} D\left(\phi_{2}, A^{\mu} ; \ell\right) \gamma^{\mu} \frac{(\ell+\not p) R-m L}{(\ell+p)^{2}-m^{2}}
\end{gathered}
$$




$$
\begin{aligned}
& \lim _{n \rightarrow 4}\left(\Gamma_{\left.\Gamma_{(e)}^{R 5}-\Gamma_{\Pi_{(e)}}^{B M}\right)}\right) \\
& =g f \int \frac{d^{n} \ell}{(2 \pi)^{n}} D\left(\phi_{2}, A^{\mu} ; \ell\right) \frac{\gamma^{\mu} \ell R-R \gamma^{\mu} L \ell \gamma_{5}}{\ell^{2}-m^{2}} \\
& =-\alpha(M-\Lambda) g f \int \frac{d^{n} \ell}{(2 \pi)^{n}} \frac{\ell_{\Delta}^{2} R}{\left(\ell^{2}-m\right)^{3}}=\frac{1}{(4 \pi)^{2}} \frac{i}{2} \alpha(M-\Lambda) f g R
\end{aligned}
$$

\section{Summary}

The amplitudes and finite counter terms due to diagrams in Figure 1 are tabulated in Table IV.

\begin{tabular}{|l|l|l|}
\hline Figure & $(4 \pi)^{2} \times\left(\Gamma^{R 5}-\Gamma^{B M}\right)$ & $(4 \pi)^{2} \times$ Counter Term \\
\hline $1(a)$ & $-i \frac{g^{2}}{3}(1+2 \alpha) \not p L$ & $-\frac{g^{2}}{3}(1+2 \alpha) \bar{\psi} R i \not \partial L \psi$ \\
$1(b)$ & 0 & 0 \\
$1(c)$ & 0 & 0 \\
$1(d)$ & $\frac{i}{2} \alpha(M-\Lambda) f g L$ & $\frac{1}{2} \alpha(M-\Lambda) f g \bar{\psi} L \psi$ \\
$1(e)$ & $\frac{i}{2} \alpha(M-\Lambda) f g R$ & $\frac{1}{2} \alpha(M-\Lambda) f g \bar{\psi} R \psi$ \\
\hline
\end{tabular}

TABLE IV. Counter terms due to diagrams in Figure 1

b. Figure 2: $\bar{\psi} A \psi$ Vertex Diagrams

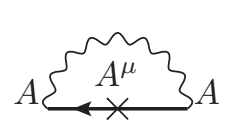

(a)

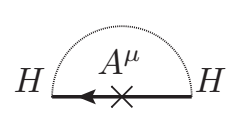

(b)

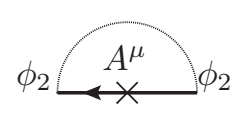

(c)

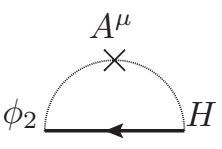

(d)

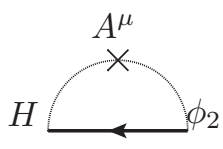

(e)

FIG. 2. Diagrams for $\bar{\psi} A \psi$ Vertex

In Figure 2, $\mu \in\{0,1,2,3\}$ is a polarization in first 4 dimensions.

Diagram (a)

$$
\Gamma_{[2}^{B M}(a)=(-i g)^{3} \int \frac{d^{n} \ell}{(2 \pi)^{n}} D\left(A_{\rho}, A_{\sigma}, \ell\right) R \gamma^{\rho} L \frac{i}{\ell+\not p+\not k-m} R \gamma^{\mu} L \frac{i}{\ell+\not p-m} R \gamma^{\sigma} L
$$




$$
\begin{aligned}
\Gamma_{[]_{a}^{R 5}}^{R 5} & =-i g^{3} \int \frac{d^{n} \ell}{(2 \pi)^{n}} D\left(A_{\rho}, A_{\sigma}, \ell\right) \gamma^{\rho} \frac{\ell+\not p+\not k}{(\ell+p+k)^{2}-m^{2}} \gamma^{\mu} \frac{\ell+\not p}{(\ell+p)^{2}-m^{2}} \gamma^{\sigma} L \\
& \Gamma_{\underline{2}_{(a)}^{R 5}}-\Gamma_{[2}^{B M}(a) \\
& =-i g^{3} \int \frac{d^{n} \ell}{(2 \pi)^{n}} D\left(A_{\rho}, A_{\sigma}, \ell\right) \frac{\gamma^{\rho} \ell \gamma^{\mu} \ell \gamma^{\sigma} L-R \gamma^{\rho} L \ell R \gamma^{\mu} L \ell R \gamma^{\sigma} L}{\left(\ell^{2}-m^{2}\right)^{2}} \\
& =-g^{3} \int \frac{d^{n} \ell}{(2 \pi)^{n}} \frac{\left(g^{\rho \sigma}+(\alpha-1) \frac{\ell^{\rho} \ell^{\sigma}}{\ell^{2}-m^{2}}\right)\left(\gamma^{\rho} \ell \gamma^{\mu} \ell \gamma^{\sigma}-\underline{\gamma}^{\rho} \underline{\ell} \gamma^{\mu} \underline{\ell \gamma^{\sigma}}\right) L}{\left(\ell^{2}-m^{2}\right)^{4}} \\
& =-g^{3} \int \frac{d^{n} \ell}{(2 \pi)^{n}} \frac{\left(\frac{(2-n)^{2}}{n} \ell^{2}-\underline{\ell}^{2}+(\alpha-1) \frac{\left(\ell^{4}-\underline{\ell}^{4}\right)}{\ell^{2}-m^{2}}\right) \gamma^{\mu} L}{\left(\ell^{2}-m^{2}\right)^{4}} \\
& =\frac{1}{(4 \pi)^{2}} \frac{i}{6} g^{3}(7+5 \alpha) \gamma^{\mu} L \quad
\end{aligned}
$$

Diagram (b)

$$
\begin{aligned}
& \Gamma_{[2}^{B M}(b)=(-i g)(-i f)^{2} \int \frac{d^{n} \ell}{(2 \pi)^{n}} D(H, H ; \ell) \frac{i}{\ell+\not p+\not k-m} R \gamma^{\mu} L \frac{i}{\ell+\not p-m} \\
& \Gamma_{[2(b)}^{R 5}=-i g f^{2} \int \frac{d^{n} \ell}{(2 \pi)^{n}} D(H, H ; \ell) \frac{1}{\ell+\not p+\not k-m} \gamma^{\mu} \frac{(\ell+\not p) R+m L}{(\ell+p)^{2}-m^{2}} \\
& \Gamma_{[2(b)}^{R 5}-\Gamma_{[2(b)}^{B M} \\
& =g f^{2} \int \frac{d^{n} \ell}{(2 \pi)^{n}} \frac{1}{\ell^{2}-m^{2}} \frac{1}{\ell-m}\left(\frac{\gamma^{\mu} \ell R}{\ell^{2}-m^{2}}-\frac{R \gamma^{\mu} L \ell \ell}{\ell^{2}-m^{2}}\right) \\
& =-g f^{2} \int \frac{d^{n} \ell}{(2 \pi)^{n}} \frac{\ell_{\Delta}^{2} \gamma_{5}}{\left(\ell^{2}-m^{2}\right)^{3}}=\frac{1}{(4 \pi)^{2}} \frac{1}{2} i g f^{2} \gamma^{\mu} \gamma_{5}
\end{aligned}
$$

Diagram (c)

$$
\begin{aligned}
& \Gamma_{\left[2{ }_{(c)}^{B M}\right.}^{\left[_{(1)}\right.}=-i g f^{2} \int \frac{d^{n} \ell}{(2 \pi)^{n}} D\left(\phi_{2}, \phi_{2} ; \ell\right) \gamma_{5} \frac{i}{\ell+\not p+\not k-m} R \gamma^{\mu} L \frac{i}{\ell+\not p-m} \gamma_{5} \\
& \Gamma_{[2}^{R 5}(c)=-i g f^{2} \int \frac{d^{n} \ell}{(2 \pi)^{n}} D\left(\phi_{2}, \phi_{2} ; \ell\right) \frac{1}{\ell+\not p+\not k+m} \gamma^{\mu} \frac{(\ell+\not p) R-m L}{(\ell+p)^{2}-m^{2}} \\
& \lim _{n \rightarrow 4}\left(\Gamma_{[2(c)}^{R 5}-\Gamma_{[2(c)}^{B M}\right) \\
& =g f^{2} \int \frac{d^{n} \ell}{(2 \pi)^{n}} \frac{1}{\ell^{2}-m^{2}}\left(\frac{1}{\ell+m} \gamma^{\mu} \frac{\ell R}{\ell^{2}-m^{2}}+\gamma_{5} \frac{1}{\ell-m} R \gamma^{\mu} L \frac{1}{\ell-m} \gamma_{5}\right) \\
& =-g f^{2} \int \frac{d^{n} \ell}{(2 \pi)^{n}} \frac{\ell_{\Delta}^{2} \gamma_{5}}{\left(\ell^{2}-m^{2}\right)^{3}}=\frac{1}{(4 \pi)^{2}} i \frac{1}{2} g f^{2} \gamma^{\mu} \gamma_{5}
\end{aligned}
$$


Diagram $(d)$

$$
\begin{aligned}
& \Gamma_{[2}^{\left.\frac{B M}{2} d\right)}=g f^{2} \int \frac{d^{n} \ell}{(2 \pi)^{n}} D\left(\phi_{2}, \phi_{2} ; \ell-k\right)(2 \ell-k)^{\mu} D(H, H ; \ell) \gamma_{5} \frac{1}{\ell+\not p-m} \\
& \Gamma_{\left.2 \_d\right)}^{R 5}=g f^{2} \int \frac{d^{n} \ell}{(2 \pi)^{n}} D\left(\phi_{2}, \phi_{2} ; \ell-k\right)(2 \ell-k)^{\mu} D(H, H ; \ell) \frac{-1}{\ell+\not p+m} \gamma_{5} \\
& \lim _{n \rightarrow 4}\left(\Gamma_{[2]}^{R 5}(d)-\Gamma_{[2(d)}^{B M}\right) \\
& =g f^{2} \int \frac{d^{n} \ell}{(2 \pi)^{n}} D\left(\phi_{2}, \phi_{2} ; \ell\right) D(H, H ; \ell) 2 \ell^{\mu}\left(\frac{-1}{\ell+m} \gamma_{5}-\gamma_{5} \frac{1}{\ell-m}\right) \\
& =-4 g f^{2} \int \frac{d^{n} \ell}{(2 \pi)^{n}} D\left(\phi_{2}, \phi_{2} ; \ell\right) D(H, H ; \ell) \frac{\ell^{\mu} \ell_{\Delta}}{\left(\ell^{2}-m^{2}\right)}=0
\end{aligned}
$$

Diagram $(e)$

$$
\begin{gathered}
\Gamma \frac{B M}{2(e)}=g f^{2} \int \frac{d^{n} \ell}{(2 \pi)^{n}} D(H, H ; \ell-k)(k-2 \ell)^{\mu} D\left(\phi_{2}, \phi_{2} ; \ell\right) \frac{1}{\ell+\not p-m} \gamma_{5} \\
\Gamma \frac{R^{25}}{2\left(_{e)}\right.}=g f^{2} \int \frac{d^{n} \ell}{(2 \pi)^{n}} D(H, H ; \ell-k)(k-2 \ell)^{\mu} D\left(\phi_{2}, \phi_{2} ; \ell\right) \frac{1}{\ell+\not p-m} \gamma_{5} \\
\Gamma \frac{R 5}{2\left(_{e)}\right.}-\Gamma \frac{B M}{\left.2 \sum_{e}\right)}=0
\end{gathered}
$$

Summary

The amplitudes and finite counter terms due to diagrams in Figure 2 are tabulated in Table V.

\begin{tabular}{|l|l|l|}
\hline Figure & $(4 \pi)^{2} \times\left(\Gamma^{R 5}-\Gamma^{B M}\right)$ & $(4 \pi)^{2} \times$ Counter Term \\
\hline 2( $(a)$ & $\frac{i}{6} g^{3}(7+5 \alpha) \gamma^{\mu} L$ & $\frac{1}{6} g^{3}(7+5 \alpha) \bar{\psi} R \quad A L \psi$ \\
\hline $2(b)$ & $\frac{1}{2} i g f^{2} \gamma^{\mu} \gamma_{5}$ & $\frac{1}{2} g f^{2} \bar{\psi} / A \gamma_{5} \psi$ \\
$2(c)$ & $\frac{1}{2} i g f^{2} \gamma^{\mu} \gamma_{5}$ & $\frac{1}{2} g f^{2} \bar{\psi} / A \gamma_{5} \psi$ \\
$2(d)$ & 0 & 0 \\
2( $(e)$ & 0 & 0 \\
\hline
\end{tabular}

TABLE V. Counter terms due to diagrams in Figure 2 


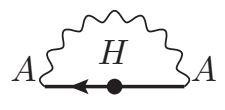

(a)

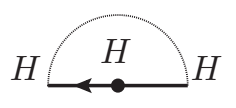

(b)

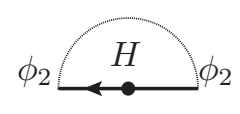

(c)

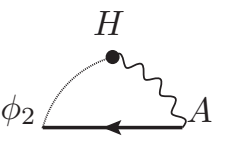

$(d)$

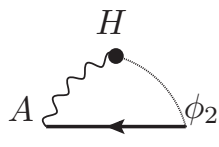

(e)

FIG. 3. Diagrams for $\bar{\psi} H \psi$ Vertex

c. Figure 3: $\bar{\psi} H \psi$ Vertex Diagrams

Diagram (a)

$$
\begin{aligned}
& \Gamma_{[3(a)}^{B M}=-i f g^{2} \int \frac{d^{n} \ell}{(2 \pi)^{n}} D\left(A_{\mu}, A_{\nu} ; \ell\right) R \gamma^{\mu} L \frac{1}{\ell+\not p+\not k-m} \frac{1}{\ell+\not p-m} R \gamma^{\nu} L \\
& \Gamma_{3(a)}^{R 5}=-i f g^{2} \int \frac{d^{n} \ell}{(2 \pi)^{n}} D\left(A_{\mu}, A_{\nu} ; \ell\right) \gamma^{\mu} \frac{m(2 \ell \ell+2 \not p+\not k)}{\left((\ell+p+k)^{2}-m^{2}\right)\left((\ell+p)^{2}-m^{2}\right)} \gamma^{\nu} L
\end{aligned}
$$

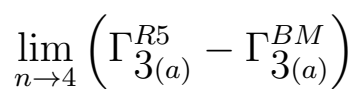

$$
\begin{aligned}
& =2 f g^{2} \int \frac{d^{n} \ell}{(2 \pi)^{n}} \frac{g_{\mu \nu}+(\alpha-1) \frac{\ell_{\mu} \ell_{\nu}}{\ell^{2}}}{\left(\ell^{2}-m^{2}\right)^{3}}\left(\begin{array}{c}
m \gamma^{\mu} \ell \gamma^{\nu} \\
-\underline{\gamma}^{\mu}\left(m+\ell_{\Delta}\right) \underline{\ell \gamma^{\nu}}
\end{array}\right) L \\
& =-2 f g^{2} \int \frac{d^{n} \ell}{(2 \pi)^{n}} \frac{g_{\mu \nu}+(\alpha-1) \frac{\ell_{\mu} \ell_{\nu}}{\ell^{2}}}{\left(\ell^{2}-m^{2}\right)^{\mu}} \underline{\gamma}^{\mu} \ell_{\Delta} \underline{\ell \gamma^{\nu}} L=0
\end{aligned}
$$

Diagram (b)

No $\gamma_{5}$ is involved.

$$
\left.\Gamma_{[3}^{R 5} b\right)-\Gamma_{[3}^{B M}(b)=0
$$

Diagram $(c)$

$$
\begin{aligned}
& \Gamma_{[3(c)}^{B M}=-i f^{3} \int \frac{d^{n} \ell}{(2 \pi)^{n}} D\left(\phi_{2}, \phi_{2} ; \ell\right) \gamma_{5} \frac{1}{\ell+\not p+\not k-m} \frac{1}{\ell+\not p-m} \gamma_{5} \\
& \Gamma_{[3(c)}^{R 5}=-i f^{3} \int \frac{d^{n} \ell}{(2 \pi)^{n}} D\left(\phi_{2}, \phi_{2} ; \ell\right) \frac{1}{\ell+\not p+\not k+m} \frac{1}{\ell+\not p+m} \\
& \lim _{n \rightarrow 4}\left(\Gamma_{[3}^{R 5}(c)-\Gamma_{[3}^{B M}(c)\right) \\
& =f^{3} \int \frac{d^{n} \ell}{(2 \pi)^{n}} \frac{1}{\left(\ell^{2}-m^{2}\right)}\left(\left(\frac{1}{\ell+m}\right)^{2}-\gamma_{5}\left(\frac{1}{\ell-m}\right)^{2} \gamma_{5}\right) \\
& =f^{3} \int \frac{d^{n} \ell}{(2 \pi)^{n}} \frac{1}{\left(\ell^{2}-m^{2}\right)^{3}}\left(\ell^{2} \gamma_{5}-\gamma_{5} \ell^{2} \gamma_{5}\right)=0
\end{aligned}
$$


$\operatorname{Diagram}(d)$

$$
\begin{aligned}
& \Gamma_{[3}^{B M}(d)=g^{2} f \int \frac{d^{n} \ell}{(2 \pi)^{n}} D\left(\phi_{2}, \phi_{2} ; \ell\right) D\left(A_{\mu}, A_{\nu} ; \ell+k\right)(\ell-k)^{\mu} \gamma_{5} \frac{1}{\ell+\not p+\not k-m} R \gamma^{\nu} L \\
& \Gamma_{[3}^{R 5}(d)=-g^{2} f \int \frac{d^{n} \ell}{(2 \pi)^{n}} D\left(\phi_{2}, \phi_{2} ; \ell\right) D\left(A_{\mu}, A_{\nu} ; \ell+k\right)(\ell-k)^{\mu} \frac{1}{\ell+\not p+\not k+m} \gamma^{\nu} L \\
& \lim _{n \rightarrow 4}\left(\Gamma_{[3]}^{R 5}(d)-\Gamma_{[3]}^{B M}(d)\right) \\
& =-g^{2} f \int \frac{d^{n} \ell}{(2 \pi)^{n}} D\left(\phi_{2}, \phi_{2} ; \ell\right) D\left(A_{\mu}, A_{\nu} ; \ell\right) \ell^{\mu}\left(\frac{1}{\ell+m} \gamma^{\nu} L+\gamma_{5} \frac{1}{\ell-m} R \gamma^{\nu} L\right) \\
& =-g^{2} f \int \frac{d^{n} \ell}{(2 \pi)^{n}} \frac{\alpha}{\left(\ell^{2}-m^{2}\right)^{3}}\left(\ell^{2} L+\gamma_{5} \ell R \ell L\right) \\
& =-g^{2} f \int \frac{d^{n} \ell}{(2 \pi)^{n}} \frac{\alpha \ell_{\Delta}^{2}}{\left(\ell^{2}-m^{2}\right)^{3}} L=\frac{1}{(4 \pi)^{2}} i \frac{\alpha g^{2} f}{2} L
\end{aligned}
$$

Diagram $(e)$

$$
\begin{aligned}
& \Gamma_{[3(e)}^{B M}=g f \int \frac{d^{n} \ell}{(2 \pi)^{n}} D\left(A_{\mu}, A_{\nu} ; \ell\right)\left(-g(\ell+2 k)^{\mu}\right) D\left(\phi_{2}, \phi_{2} ; \ell+k\right) R \gamma^{\nu} L \frac{1}{\ell+\not p+\not k-m} \gamma_{5} \\
& \Gamma_{[3}^{R 5}(e)=g f \int \frac{d^{n} \ell}{(2 \pi)^{n}} D\left(A_{\mu}, A_{\nu} ; \ell\right)\left(-g(\ell+2 k)^{\mu}\right) D\left(\phi_{2}, \phi_{2} ; \ell+k\right) \gamma^{\nu} \frac{(\ell+\not p+\not k) R-m L}{(\ell+p+k)^{2}-m^{2}} \\
& \lim _{n \rightarrow 4}\left(\Gamma_{[3]}^{R 5}(e)-\Gamma_{[3(e)}^{B M}\right) \\
& =-g^{2} f \int \frac{d^{n} \ell}{(2 \pi)^{n}} \frac{\alpha \ell_{\Delta}^{2}}{\left(\ell^{2}-m^{2}\right)^{3}}\left(\ell^{2} R-R \ell L \ell \gamma_{5}\right) \\
& =-g^{2} f \int \frac{d^{n} \ell}{(2 \pi)^{n}} \frac{\alpha \ell_{\Delta}^{2}}{\left(\ell^{2}-m^{2}\right)^{3}} R=\frac{1}{(4 \pi)^{2}} i \frac{\alpha g^{2} f}{2} R
\end{aligned}
$$

Summary

The amplitudes and finite counter terms due to diagrams in Figure 3 are tabulated in Table VI.

d. Figure 4: $\bar{\psi} \phi_{2} \psi$ Vertex Diagrams

Diagram (a)

$$
\Gamma_{[4}^{B M}(a)=f g^{2} \int \frac{d^{n} \ell}{(2 \pi)^{n}} D\left(A_{\mu}, A_{\nu} ; \ell\right) R \gamma^{\mu} L \frac{1}{\ell+\not p+\not k-m} \gamma_{5} \frac{1}{\ell+\not p-m} R \gamma^{\nu} L
$$




\begin{tabular}{|l|l|l|}
\hline Figure & $(4 \pi)^{2} \times\left(\Gamma^{R 5}-\Gamma^{B M}\right)$ & $(4 \pi)^{2} \times$ Counter Term \\
\hline $3(a)$ & 0 & 0 \\
\hline $3(b)$ & 0 & 0 \\
$3(c)$ & 0 & 0 \\
$3(d)$ & $i \frac{\alpha g^{2} f}{2} L$ & $\frac{\alpha g^{2} f}{2} \bar{\psi} H L \psi$ \\
$3(e)$ & $i \frac{\alpha g^{2} f}{2} R$ & $\frac{\alpha g^{2} f}{2} \bar{\psi} H R \psi$ \\
\hline
\end{tabular}

TABLE VI. Counter terms due to diagrams in Figure 3

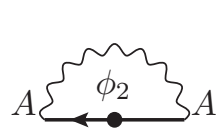

(a)

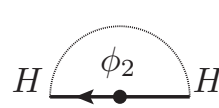

(b)

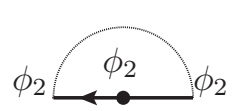

$(c)$

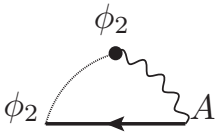

$(d)$

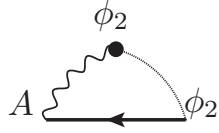

$(e)$

FIG. 4. Diagrams for $\bar{\psi} \phi_{2} \psi$ Vertex

$$
\begin{aligned}
& \Gamma_{\Psi_{(a)}^{R 5}}^{R(}=f g^{2} \int \frac{d^{n} \ell}{(2 \pi)^{n}} D\left(A_{\mu}, A_{\nu} ; \ell\right) \gamma^{\mu} \frac{m \not k}{\left((\ell+p+k)^{2}-m^{2}\right)\left((\ell+p)^{2}-m^{2}\right)} \gamma^{\nu} L \\
& \lim _{n \rightarrow 4}\left(\Gamma_{[4}^{R 5}(a)-\Gamma_{4}^{B M}(a)\right) \\
& =-i f g^{2} \int \frac{d^{n} \ell}{(2 \pi)^{n}} \frac{g_{\mu \nu}+(\alpha-1) \frac{\ell_{\mu} \ell_{\nu}}{\ell^{2}}}{\left(\ell^{2}-m^{2}\right)^{3}}\left(-2 m \underline{\gamma}^{\mu} \ell_{\Delta} \underline{\gamma}^{\nu}\right) L=0
\end{aligned}
$$

Diagram (b)

$$
\begin{aligned}
& \Gamma_{[4(b)}^{B M}=f^{3} \int \frac{d^{n} \ell}{(2 \pi)^{n}} D\left(\phi_{2}, \phi_{2} ; \ell\right) \frac{1}{\ell+\not p+\not k-m} \gamma_{5} \frac{1}{\ell+\not p-m} \\
& \Gamma_{[4(b)}^{R 5}=-f^{3} \int \frac{d^{n} \ell}{(2 \pi)^{n}} D\left(\phi_{2}, \phi_{2} ; \ell\right) \frac{1}{\ell+\not p+\not k-m} \frac{1}{\ell+\not p+m} \gamma_{5} \\
& \lim _{n \rightarrow 4}\left(\Gamma_{[4}^{R 5}(b)-\Gamma_{[4}^{B M} b\right) \\
& =-i f^{3} \int \frac{d^{n} \ell}{(2 \pi)^{n}} \frac{1}{\left(\ell^{2}-m^{2}\right)}\left(\frac{1}{\left(\ell^{2}-m^{2}\right)} \gamma_{5}+\frac{1}{\ell-m} \gamma_{5} \frac{1}{\ell-m}\right) \\
& =-i f^{3} \int \frac{d^{n} \ell}{(2 \pi)^{n}} \frac{1}{\left(\ell^{2}-m^{2}\right)^{2}}\left(\gamma_{5}+\frac{1}{\left(\ell^{2}-m^{2}\right)} \ell \gamma_{5} \ell\right) \\
& =-2 i f^{3} \int \frac{d^{n} \ell}{(2 \pi)^{n}} \frac{\ell_{\Delta}^{2}}{\left(\ell^{2}-m^{2}\right)^{3}} \gamma_{5}=-\frac{1}{(4 \pi)^{2}} f^{3} \gamma_{5}
\end{aligned}
$$


Diagram $(c)$

$$
\begin{aligned}
& \Gamma_{[4(c)}^{B M}=-f^{3} \int \frac{d^{n} \ell}{(2 \pi)^{n}} D\left(\phi_{2}, \phi_{2} ; \ell\right) \gamma_{5} \frac{1}{\ell+\not p+\not k-m} \gamma_{5} \frac{1}{\ell+\not p-m} \gamma_{5} \\
& \Gamma_{[4}^{R 5}(c)=f^{3} \int \frac{d^{n} \ell}{(2 \pi)^{n}} D\left(\phi_{2}, \phi_{2} ; \ell\right) \frac{1}{\ell+\not p+\not k+m} \frac{1}{\ell+\not p-m} \gamma_{5} \\
& \lim _{n \rightarrow 4}\left(\Gamma_{[4(c)}^{R 5}-\Gamma_{[4}^{B M}(c)\right) \\
& =i f^{3} \int \frac{d^{n} \ell}{(2 \pi)^{n}} \frac{1}{\left(\ell^{2}-m^{2}\right)^{2}}\left(\gamma_{5}+\frac{1}{\left(\ell^{2}-m^{2}\right)} \gamma_{5} \ell \gamma_{5} \ell \gamma_{5}\right) \\
& =2 i f^{3} \int \frac{d^{n} \ell}{(2 \pi)^{n}} \frac{\ell_{\Delta}^{2}}{\left(\ell^{2}-m^{2}\right)^{3}} \gamma_{5}=\frac{1}{(4 \pi)^{2}} f^{3} \gamma_{5}
\end{aligned}
$$

Diagram $(d)$

$$
\begin{aligned}
& \Gamma_{[4(d)}^{B M}=-i f g^{2} \int \frac{d^{n} \ell}{(2 \pi)^{n}} D(H, H ; \ell)(k-\ell)^{\mu} D\left(A_{\mu}, A_{\nu} ; \ell+k\right) \frac{1}{\ell+\not p+\not k-m} R \gamma^{\nu} L \\
& \Gamma_{\Psi_{(d)}^{R 5}}^{(d)}=-i f g^{2} \int \frac{d^{n} \ell}{(2 \pi)^{n}} D(H, H ; \ell)(k-\ell)^{\mu} D\left(A_{\mu}, A_{\nu} ; \ell+k\right) \frac{1}{\ell+\not p+\not k-m} \gamma^{\nu} L \\
& \lim _{n \rightarrow 4}\left(\Gamma_{[4(d)}^{R 5}-\Gamma_{[4}^{B M}(d)\right) \\
& =-i f g \int \frac{d^{n} \ell}{(2 \pi)^{n}} D(H, H ; \ell) g(k-\ell)^{\mu} D\left(A_{\mu}, A_{\nu} ; \ell+k\right) \frac{1}{\ell-m}\left(\gamma^{\nu} L-R \gamma^{\nu} L\right) \\
& =i f g^{2} \int \frac{d^{n} \ell}{(2 \pi)^{n}} \frac{\alpha \ell_{\Delta}^{2}}{\left(\ell^{2}-m^{2}\right)^{3}} L=\frac{1}{(4 \pi)^{2}} \frac{1}{2} \alpha f g^{2} L
\end{aligned}
$$

$\operatorname{Diagram}(e)$

$$
\begin{aligned}
& \Gamma_{[4(e)}^{B M}=-i g f \int \frac{d^{n} \ell}{(2 \pi)^{n}} D\left(A_{\mu}, A_{\nu} ; \ell\right) g(\ell+2 k)^{\nu} D(H, H ; \ell+k) R \gamma^{\mu} L \frac{1}{\ell+\not p+\not k-m} \\
& \Gamma_{[4}^{R 5}(e)=-i g f \int \frac{d^{n} \ell}{(2 \pi)^{n}} D\left(A_{\mu}, A_{\nu} ; \ell\right) g(\ell+2 k)^{\nu} D(H, H ; \ell+k) \gamma^{\mu} \frac{(\ell+\not p+\not k) R+m L}{(\ell+p+k)^{2}-m^{2}} \\
& \lim _{n \rightarrow 4}\left(\Gamma_{[4(e)}^{R 5}-\Gamma_{[4}^{B M}(e)\right) \\
& =-i g f \int \frac{d^{n} \ell}{(2 \pi)^{n}} D\left(A_{\mu}, A_{\nu} ; \ell\right) g \ell^{\nu} D(H, H ; \ell)\left(\gamma^{\mu} \frac{\ell R}{\ell^{2}-m^{2}}-R \gamma^{\mu} L \frac{1}{\ell-m}\right) \\
& =-i g^{2} f \int \frac{d^{n} \ell}{(2 \pi)^{n}} \frac{\alpha \ell_{\Delta}^{2}}{\left(\ell^{2}-m^{2}\right)^{3}} R=-\frac{1}{(4 \pi)^{2}} \frac{\alpha g^{2} f}{2} R
\end{aligned}
$$

Summary

The amplitudes and finite counter terms due to diagrams in Figure 4 are tabulated in Table VII. 


\begin{tabular}{|l|l|l|}
\hline Figure & $(4 \pi)^{2} \times\left(\Gamma^{R 5}-\Gamma^{B M}\right)$ & $(4 \pi)^{2} \times$ Counter Term \\
\hline $4(a)$ & 0 & 0 \\
\hline $4(b)$ & $-f^{3} \gamma_{5}$ & $i f^{3} \bar{\psi} \phi_{2} \gamma_{5} \psi$ \\
$4(c)$ & $f^{3} \gamma_{5}$ & $-i f^{3} \bar{\psi} \phi_{2} \gamma_{5} \psi$ \\
$4(d)$ & $\frac{\alpha g^{2} f}{2} L$ & $-i \frac{\alpha g^{2} f}{2} \bar{\psi} \phi_{2} L \psi$ \\
$4(e)$ & $-\frac{\alpha g^{2} f}{2} R$ & $i \frac{\alpha g^{2} f}{2} \bar{\psi} \phi_{2} R \psi$ \\
\hline
\end{tabular}

TABLE VII. Counter terms due to diagrams in Figure 4

e. Figure 5: One-Fermion-Loop 2-Point 1PI

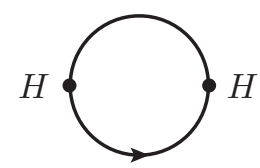

(a)

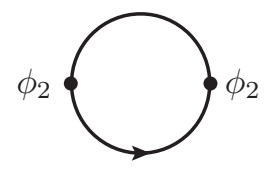

(b)

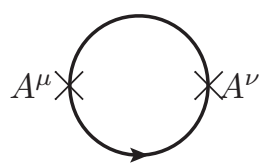

(c)

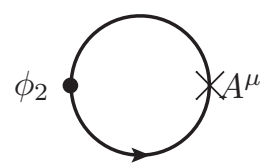

(d)

FIG. 5. Diagrams for 2-point 1PI

Diagram $(a)$

No $\gamma_{5}$ occurs in the amplitude. Thus,

$$
\Gamma_{5(a)}^{R 5}-\Gamma_{5](a)}^{B M}=0
$$

Diagram (b)

$$
\begin{gathered}
\Gamma_{[5(b)}^{B M}=-f^{2} \operatorname{tr} \int \frac{d^{n} \ell}{(2 \pi)^{n}} \frac{i}{\ell-m} \gamma_{5} \frac{i}{\ell-\not p-m} \gamma_{5} \\
\Gamma_{[5(b)}^{R 5}=f^{2} \operatorname{tr} \int \frac{d^{n} \ell}{(2 \pi)^{n}} \frac{i}{\ell-m} \frac{i}{\ell-\not p+m}
\end{gathered}
$$




$$
\begin{aligned}
& \lim _{n \rightarrow 4}\left(\Gamma_{[55}^{R 5}-\Gamma_{[5(b)}^{B M}\right) \\
& =-f^{2} \operatorname{tr} \int \frac{d^{n} \ell}{(2 \pi)^{n}} \frac{1}{\ell-m}\left(\frac{1}{\ell-\not p+m}+\gamma_{5} \frac{1}{\ell-\not p-m} \gamma_{5}\right) \\
& =-f^{2} \int \frac{d^{n} \ell}{(2 \pi)^{n}} \frac{\operatorname{tr}\left[(\ell+m) 2 \ell_{\Delta}\right]}{\left(\ell^{2}-m^{2}\right)\left((\ell-p)^{2}-m^{2}\right)} \\
& =-8 f^{2} \int \frac{d^{n} \ell}{(2 \pi)^{n}} \ell_{\Delta}^{2}\left(\frac{1}{\left(\ell^{2}-m^{2}\right)^{2}}+\frac{2 \ell \cdot p-p^{2}}{\left(\ell^{2}-m^{2}\right)^{3}}+\frac{\left(2 \ell \cdot p-p^{2}\right)^{2}}{\left(\ell^{2}-m^{2}\right)^{4}}\right) \\
& =\frac{1}{(4 \pi)^{2}} i f^{2}\left(8 m^{2}-\frac{4}{3} p^{2}\right)
\end{aligned}
$$

Diagram $(c)$

$$
\begin{aligned}
& \Gamma_{[5(c)}^{B M}=-g^{2} \operatorname{tr} \int \frac{d^{n} \ell}{(2 \pi)^{n}} \frac{1}{\ell-m} R \gamma^{\mu} L \frac{1}{\ell-\not p-m} R \gamma^{\nu} L \\
& \Gamma_{[5]}^{R 5}=-g^{2} \operatorname{tr} \int \frac{d^{n} \ell}{(2 \pi)^{n}} \frac{1}{\ell-m} \gamma^{\mu} \frac{\ell-\not p}{(\ell-p)^{2}-m^{2}} \gamma^{\nu} L \\
& \lim _{n \rightarrow 4}\left(\Gamma_{[5]}^{R 5}-\Gamma_{[5]}^{B M}(c)\right) \\
& =-g^{2} \operatorname{tr} \int \frac{d^{n} \ell}{(2 \pi)^{n}} \frac{1}{\ell-m} \gamma^{\mu}\left(\frac{\ell-L \ell R}{(\ell-p)^{2}-m^{2}}\right) \gamma^{\nu} L \\
& =2 g^{\mu \nu} g^{2} \int \frac{d^{n} \ell}{(2 \pi)^{n}} \ell_{\Delta}^{2}\left(\frac{1}{\left(\ell^{2}-m^{2}\right)^{2}}+\frac{2 \ell \cdot p-p^{2}}{\left(\ell^{2}-m^{2}\right)^{3}}+\frac{\left(2 \ell \cdot p-p^{2}\right)^{2}}{\left(\ell^{2}-m^{2}\right)^{4}}\right) \\
& =\frac{1}{(4 \pi)^{2}} i g^{\mu \nu} g^{2}\left(-2 m^{2}+\frac{1}{3} p^{2}\right)
\end{aligned}
$$

$\operatorname{Diagram}(d)$

$$
\begin{aligned}
& \Gamma_{5](d)}^{B M}=-i f g \int \frac{d^{n} \ell}{(2 \pi)^{n}} \operatorname{tr}\left(\frac{1}{\ell-\not p-m} \gamma^{5} \frac{1}{\ell-m} R \gamma^{\mu} L\right) \\
& \Gamma_{5_{(d)}^{R 5}}=-i f g \int \frac{d^{n} \ell}{(2 \pi)^{n}} \operatorname{tr}\left(\frac{1}{\ell-\not p-m} \frac{1}{-\ell-m} \gamma^{\mu} L\right) \\
& \lim _{n \rightarrow 4}\left(\Gamma_{[5]}^{R 5}-\Gamma_{[5]}^{B M}(d)\right) \\
& =i f g \int \frac{d^{n} \ell}{(2 \pi)^{n}} \operatorname{tr}\left(\frac{1}{\ell-\not p-m}\left(\frac{1}{\ell+m}+\gamma^{5} \frac{1}{\ell-m} R\right) \gamma^{\mu} L\right) \\
& =i f g \int \frac{d^{n} \ell}{(2 \pi)^{n}} \operatorname{tr}\left(\frac{2(\ell-\not p+m) \ell_{\Delta} \gamma^{\mu} L}{\left((\ell-p)^{2}-m^{2}\right)\left(\ell^{2}-m^{2}\right)}\right)=0
\end{aligned}
$$

Summary

The amplitudes and finite counter terms due to diagrams in Figure 5 are tabulated in Table VIII. 


\begin{tabular}{|l|l|l|}
\hline Figure & $(4 \pi)^{2} \times\left(\Gamma^{R 5}-\Gamma^{B M}\right)$ & $(4 \pi)^{2} \times$ Counter Term \\
\hline $5(a)$ & 0 & 0 \\
$5(b)$ & $i f^{2}\left(8 m^{2}-\frac{4}{3} p^{2}\right)$ & $4 m^{2} f^{2}\left(\phi_{2}\right)^{2}-\frac{2}{3} f^{2}\left(\partial_{\mu} \phi_{2}\right)\left(\partial^{\mu} \phi_{2}\right)$ \\
$5(c)$ & $i g^{\mu \nu} g^{2}\left(-2 m^{2}+\frac{1}{3} p^{2}\right)$ & $-g^{2} m^{2} A^{2}+\frac{1}{6} g^{2}\left(\partial_{\mu} A_{\nu}\right)\left(\partial^{\mu} A^{\nu}\right)$ \\
$5(d)$ & 0 & 0 \\
\hline
\end{tabular}

TABLE VIII. Counter terms due to diagrams in Figure 5

\section{f. Figure [6: One-Fermion-Loop 3-Point 1PI}

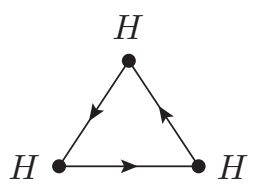

(a)

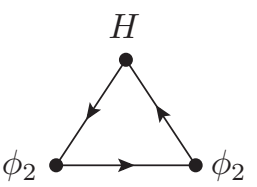

(b)

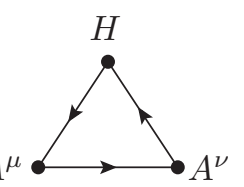

(c)

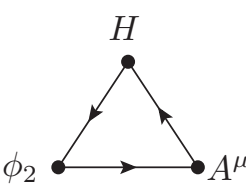

(d)

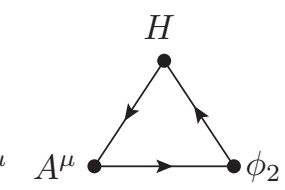

(e)

FIG. 6. Diagrams for 3-point 1PI

Diagram $(a)$

No $\gamma_{5}$ is involved and therefore there is no finite counter term.

Diagram $(b)$

$$
\begin{gathered}
\Gamma_{[\underline{6} b)}^{B M}=f^{3} \operatorname{tr} \int \frac{d^{n} \ell}{(2 \pi)^{n}} \frac{1}{\ell-m} \frac{1}{\ell-\not p_{1}-m} \gamma_{5} \frac{1}{\ell-\not p_{1}-\not p_{2}-m} \gamma_{5} \\
\Gamma_{[\underline{6}(b)}^{R 5}=-f^{3} \operatorname{tr} \int \frac{d^{n} \ell}{(2 \pi)^{n}} \frac{1}{\ell-m} \frac{1}{\ell-\not p_{1}-m} \frac{1}{\ell-\not p_{1}-\not p_{2}+m} \\
\lim _{n \rightarrow 4}\left(\Gamma_{[\underline{6}(b)}^{R 5}-\Gamma_{[\underline{6}(b)}^{B M}\right) \\
=f^{3} \operatorname{tr} \int \frac{d^{n} \ell}{(2 \pi)^{n}} \frac{1}{\ell-m} \frac{1}{\ell-\not p_{1}-m} \frac{\left(-\ell+\not p_{1}+\not p_{2}+m\right)-\gamma_{5}\left(\ell-\not p_{1}-\not p_{2}+m\right) \gamma_{5}}{\left(\ell-\not p_{1}-\not p_{2}\right)^{2}-m^{2}} \\
=-2 f^{3} \operatorname{tr} \int \frac{d^{n} \ell}{(2 \pi)^{n}} \frac{(\ell+m)\left(\ell-\not p_{1}+m\right) \not \ell_{\Delta}}{\left(\ell^{2}-m^{2}\right)\left(\left(\ell-p_{1}\right)^{2}-m^{2}\right)\left(\left(\ell-p_{1}-p_{2}\right)^{2}-m^{2}\right)} \\
=-2 f^{3} \operatorname{tr} \int \frac{d^{n} \ell}{(2 \pi)^{n}} \frac{\left(\not p_{1}+2 m\right) \ell_{\Delta}^{2}}{\left(\ell^{2}-m^{2}\right)^{3}}=\frac{1}{(4 \pi)^{2}} 8 i f^{3} m
\end{gathered}
$$

There is another diagram corresponding to the exchange of the two $\phi_{2}$ fields or the reverse of fermion-loop direction which also yields the same amplitude $\frac{1}{(4 \pi)^{2}} 8 i f^{3} \mathrm{~m}$. 
Diagram $(c)$

$$
\begin{aligned}
& \Gamma_{\left[{ }_{c}{ }_{c}\right)}^{B M}=-f g^{2} \operatorname{tr} \int \frac{d^{n} \ell}{(2 \pi)^{n}} \frac{1}{\ell-m} \frac{1}{\ell-\not p_{1}-m} R \gamma^{\mu} L \frac{1}{\ell-\not p_{1}-\not p_{2}-m} R \gamma^{\nu} L \\
& \Gamma_{\left[b_{c}\right)}^{R 5}=-f g^{2} \operatorname{tr} \int \frac{d^{n} \ell}{(2 \pi)^{n}} \frac{1}{\ell-m} \frac{1}{\ell-\not p_{1}-m} \gamma^{\mu} \frac{\ell-\not p_{1}-\not p_{2}}{\left(\ell-\not p_{1}-\not p_{2}\right)^{2}-m^{2}} \gamma^{\nu} L \\
& \lim _{n \rightarrow 4}\left(\Gamma_{[\underline{6}(c)}^{R 5}-\Gamma_{[\underline{6}(c)}^{B M}\right) \\
& =-f g^{2} \int \frac{d^{n} \ell}{(2 \pi)^{n}} \frac{\operatorname{tr}\left[(\ell+m)\left(\ell-\not p_{1}+m\right) \gamma^{\mu} \ell_{\Delta} \gamma^{\nu} L\right]}{\left(\ell^{2}-m^{2}\right)\left(\left(\ell-p_{1}\right)^{2}-m^{2}\right)\left(\left(\ell-p_{1}-p_{2}\right)^{2}-m^{2}\right)} \\
& =4 f g^{2} \int \frac{d^{n} \ell}{(2 \pi)^{n}} \frac{\ell_{\Delta}^{2} m g^{\mu \nu}}{\left(\ell^{2}-m^{2}\right)^{3}}=-\frac{1}{(4 \pi)^{2}} 2 i f g^{2} m g^{\mu \nu}
\end{aligned}
$$

Interchanging the two external $A$ fields gives us another topologically different diagram whose amplitude is also equal to $-\frac{1}{(4 \pi)^{2}} 2 i g^{2} f m g^{\mu \nu}$.

Diagram $(d)$

$$
\begin{aligned}
& \Gamma_{[\underline{G}(d)}^{B M}=-i f^{2} g \operatorname{tr} \int \frac{d^{n} \ell}{(2 \pi)^{n}} \frac{1}{\ell-m} \frac{1}{\ell-\not p_{1}-m} \gamma_{5} \frac{1}{\ell-\not p_{1}-\not p_{2}-m} R \gamma^{\nu} L \\
& \Gamma_{[\underline{6}[d)}^{R 5}=i f^{2} g \operatorname{tr} \int \frac{d^{n} \ell}{(2 \pi)^{n}} \frac{1}{\ell-m} \frac{1}{\ell-\not p_{1}-m} \frac{1}{\ell-\not p_{1}-\not p_{2}+m} R \gamma^{\nu} L \\
& \lim _{n \rightarrow 4}\left(\Gamma_{[6] d)}^{R 5}-\Gamma_{[6] d)}^{B M}\right) \\
& =i f^{2} g \int \frac{d^{n} \ell}{(2 \pi)^{n}} \frac{2 \operatorname{tr}\left[(\ell+m)\left(\ell-\not p_{1}+m\right) \ell_{\Delta} \gamma^{\nu} L\right]}{\left(\ell^{2}-m^{2}\right)\left(\left(\ell-p_{1}\right)^{2}-m^{2}\right)\left(\left(\ell-p_{1}-p_{2}\right)^{2}-m^{2}\right)} \\
& =i f^{2} g \int \frac{d^{n} \ell}{(2 \pi)^{n}} \frac{\ell_{\Delta}^{2}}{\left(\ell^{2}-m^{2}\right)^{3}} \operatorname{tr}\left[\not p_{1} \gamma^{\nu}\right]=\frac{1}{(4 \pi)^{2}} 2 f^{2} g p_{1}^{\nu}
\end{aligned}
$$

Diagram $(e)$

$$
\begin{aligned}
& \Gamma_{[\underline{6}(e)}^{B M}=-i f^{2} g \operatorname{tr} \int \frac{d^{n} \ell}{(2 \pi)^{n}} \frac{1}{\ell-m} \frac{1}{\ell-\not p_{1}-m} R \gamma^{\nu} L \frac{1}{\ell-\not p_{1}-\not p_{2}-m} \gamma_{5} \\
& \Gamma_{\left[{ }_{(E)}\right.}^{R 5}=-i f^{2} g \operatorname{tr} \int \frac{d^{n} \ell}{(2 \pi)^{n}} \frac{1}{\ell-m} \frac{1}{\ell-\not p_{1}-m} \gamma^{\nu} \frac{\left(\ell-\not p_{1}-\not p_{2}\right) R-m L}{\left(\left(\ell-p_{1}-p_{2}\right)^{2}-m^{2}\right)} \\
& \lim _{n \rightarrow 4}\left(\Gamma_{[\underline{6}(e)}^{R 5}-\Gamma_{[6(e)}^{B M}\right) \\
& -i f^{2} g \int \frac{d^{n} \ell}{(2 \pi)^{n}} \frac{\operatorname{tr}\left[(\ell+m)\left(\ell-\not p_{1}+m\right) \gamma^{\nu} \ell_{\Delta}\right]}{\left(\ell^{2}-m^{2}\right)\left(\left(\ell-p_{1}\right)^{2}-m^{2}\right)\left(\left(\ell-p_{1}-p_{2}\right)^{2}-m^{2}\right)} \\
& =i f^{2} g \int \frac{d^{n} \ell}{(2 \pi)^{n}} \frac{\ell_{\Delta}^{2}}{\left(\ell^{2}-m^{2}\right)^{3}} \operatorname{tr}\left[\not p_{1} \gamma^{\nu}\right]=\frac{1}{(4 \pi)^{2}} 2 f^{2} g p_{1}^{\nu}
\end{aligned}
$$




\section{Summary}

The amplitudes and finite counter terms due to diagrams in Figure 6 are tabulated in Table IX. Note the column "Multiplicity" indicates the combinatorial factor that needs to be multiplied.

\begin{tabular}{|l|l|l|l|}
\hline Figure & $(4 \pi)^{2} \times\left(\Gamma^{R 5}-\Gamma^{B M}\right)$ & Multiplicity & $(4 \pi)^{2} \times$ Counter Term \\
\hline $6(a)$ & 0 & 2 & 0 \\
\hline $6(b)$ & $8 i f^{3} m$ & 2 & $8 f^{3} m H\left(\phi_{2}\right)^{2}$ \\
$6(c)$ & $-2 i f g^{2} m g^{\mu \nu}$ & 2 & $-2 f g^{2} m H A^{2}$ \\
$6(d)$ & $2 f^{2} g p_{1}^{\nu}$ & 1 & $2 f^{2} g \phi_{2}\left(\partial_{\mu} H\right) A^{\mu}$ \\
$6(e)$ & $2 f^{2} g p_{1}^{\nu}$ & 1 & $2 f^{2} g \phi_{2}\left(\partial_{\mu} H\right) A^{\mu}$ \\
\hline
\end{tabular}

TABLE IX. Counter terms due to diagrams in Figure 6

g. Figure 7;: One-Fermion-Loop 4-Point 1PI

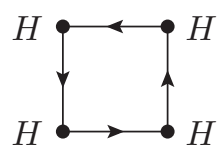

(a)

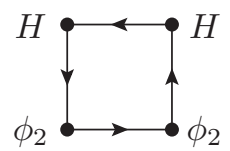

$(b)$

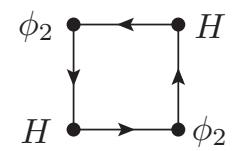

(c)

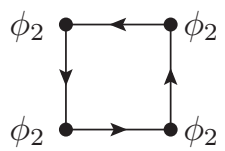

$(d)$

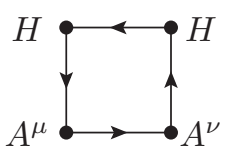

(e)

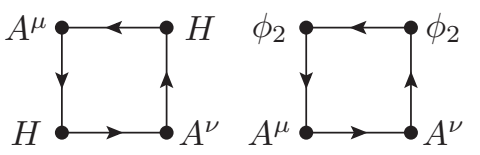

$(f)$ $(g)$

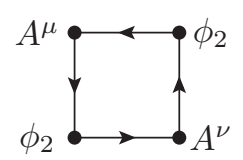

$(h)$

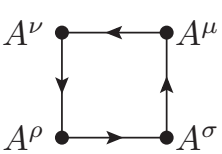

(i)

FIG. 7. Diagrams for 4-point 1PI

Only $T_{0}$ order terms may be divergent. For the sake of simplicity, we may assume all the external momenta are zero.

Diagram $(a)$

No $\gamma_{5}$ is involved and therefore diagram $(a)$ generates no finite counter term. 
Diagram $(b)$

$$
\begin{gathered}
\Gamma_{[7(b)}^{B M}=f^{4} \operatorname{tr} \int \frac{d^{n} \ell}{(2 \pi)^{n}} \frac{1}{\ell-m} \frac{1}{\ell-m} \frac{1}{\ell-m} \gamma_{5} \frac{1}{\ell-m} \gamma_{5} \\
\Gamma_{7(b)}^{R 5}=f^{4} \operatorname{tr} \int \frac{d^{n} \ell}{(2 \pi)^{n}} \frac{1}{\ell-m} \frac{1}{\ell-m} \frac{1}{\ell-m} \frac{-1}{\ell+m} \\
\lim _{n \rightarrow 4}\left(\Gamma_{77(b)}^{R 5}-\Gamma_{[7(b)}^{B M}\right)=f^{4} \lim _{n \rightarrow 4} \operatorname{tr} \int \frac{d^{n} \ell}{(2 \pi)^{n}} \frac{\ell \ell \ell}{\left(\ell^{2}-m^{2}\right)^{4}}\left(-\ell-\gamma_{5} \ell \gamma_{5}\right) \\
=-2 f^{4} \lim _{n \rightarrow 4} \int \frac{d^{n} \ell}{(2 \pi)^{n}} \frac{t r\left[\ell^{2} \ell_{\Delta}^{2}\right]}{\left(\ell^{2}-m^{2}\right)^{4}}=\frac{1}{(4 \pi)^{2}} 4 i f^{4}
\end{gathered}
$$

Exchanging the two $H$ and the two $\phi_{2}$ gives a total counter-term amplitude of $\frac{1}{(4 \pi)^{2}} 4 \times 4 i f^{4}=$ $\frac{1}{(4 \pi)^{2}} 16 i f^{4}$.

Diagram $(c)$

$$
\begin{aligned}
& \Gamma_{Z_{(c)}^{B M}}^{B M}=f^{4} \operatorname{tr} \int \frac{d^{n} \ell}{(2 \pi)^{n}} \frac{1}{\ell-m} \frac{1}{\ell-m} \gamma_{5} \frac{1}{\ell-m} \frac{1}{\ell-m} \gamma_{5} \\
& \Gamma_{[7 c)}^{R 5}=f^{4} \operatorname{tr} \int \frac{d^{n} \ell}{(2 \pi)^{n}} \frac{1}{\ell-m} \frac{1}{\ell-m} \frac{1}{\ell+m} \frac{1}{\ell+m} \\
& \lim _{n \rightarrow 4}\left(\Gamma_{\left[Z_{c}^{R}\right)}^{R 5}-\Gamma_{Z_{c}}^{B M}\right)=f^{4} \operatorname{tr} \int \frac{d^{n} \ell}{(2 \pi)^{n}} \frac{\ell \ell \ell \ell-\ell \ell \gamma_{5} \ell \ell \gamma_{5}}{\left(\ell^{2}-m^{2}\right)^{4}}=0
\end{aligned}
$$

Diagram $(d)$

$$
\begin{aligned}
& \Gamma_{7_{d d}^{B M}}^{B M}=-f^{4} \operatorname{tr} \int \frac{d^{n} \ell}{(2 \pi)^{n}} \frac{1}{\ell-m} \gamma_{5} \frac{1}{\ell-m} \gamma_{5} \frac{1}{\ell-m} \gamma_{5} \frac{1}{\ell-m} \gamma_{5} \\
& \Gamma_{7^{R}(d)}^{R 5}=-f^{4} \operatorname{tr} \int \frac{d^{n} \ell}{(2 \pi)^{n}} \frac{1}{\ell-m} \frac{1}{\ell+m} \frac{1}{\ell-m} \frac{1}{\ell+m} \\
& \lim _{n \rightarrow 4}\left(\Gamma_{[7(d)}^{R 5}-\Gamma_{[7(d)}^{B M}\right)=-f^{4} \int \frac{d^{n} \ell}{(2 \pi)^{n}} \frac{\operatorname{tr}\left[\ell^{4}-\ell \gamma_{5} \ell \gamma_{5} \ell \gamma_{5} \ell \gamma_{5}\right]}{\left(\ell^{2}-m^{2}\right)^{4}} \\
& =-32 f^{4} \int \frac{d^{n} \ell}{(2 \pi)^{n}} \frac{\ell_{\Delta}^{2} \underline{\ell}^{2}}{\left(\ell^{2}-m^{2}\right)^{4}}=\frac{1}{(4 \pi)^{2}} \frac{32}{3} i f^{4}
\end{aligned}
$$

Permutation of the four $\phi_{2}$ on a loop gives a combinatorial factor of 3!. The total counterterm amplitude due to this type of diagram is $3 ! \times \frac{1}{(4 \pi)^{2}} \frac{32}{3} i f^{4}=\frac{1}{(4 \pi)^{2}} 64 i f^{4}$ Diagram $(e)$

$$
\begin{aligned}
\Gamma_{7(e)}^{B M} & =-f^{2} g^{2} \operatorname{tr} \int \frac{d^{n} \ell}{(2 \pi)^{n}} \frac{1}{\ell-m} \frac{1}{\ell-m} \frac{1}{\ell-m} R \gamma^{\mu} L \frac{1}{\ell-m} R \gamma^{\nu} L \\
\Gamma_{7(e)}^{R 5} & =-f^{2} g^{2} \operatorname{tr} \int \frac{d^{n} \ell}{(2 \pi)^{n}} \frac{1}{\ell-m} \frac{1}{\ell-m} \frac{1}{\ell-m} \gamma^{\mu} \frac{\ell}{\left(\ell^{2}-m^{2}\right)} \gamma^{\nu} L
\end{aligned}
$$




$$
\begin{aligned}
\lim _{n \rightarrow 4}\left(\Gamma_{\left[{ }_{(e)}^{R 5}\right.}^{R 5}-\Gamma_{[7(e)}^{B M}\right) & =-f^{2} g^{2} \int \frac{d^{n} \ell}{(2 \pi)^{n}} \frac{\operatorname{tr}\left[\ell^{2} \ell \gamma^{\mu} \ell \gamma^{\nu} L-\ell^{2} \ell \gamma^{\mu} \underline{\ell} \gamma^{\nu} L\right]}{\left(\ell^{2}-m^{2}\right)^{4}} \\
& =-f^{2} g^{2} \int \frac{d^{n} \ell}{(2 \pi)^{n}} \frac{\operatorname{tr}\left[\ell \gamma^{\mu} \ell \gamma^{\nu} L\right]}{\left(\ell^{2}-m^{2}\right)^{3}}=-\frac{1}{(4 \pi)^{2}} i f^{2} g^{2} g^{\mu \nu}
\end{aligned}
$$

Exchanges of the two $H$ and of the two $A$ multiply the above amplitude by a factor of 4 . i.e., the total amplitude is $-\frac{1}{(4 \pi)^{2}} i 4 f^{2} g^{2} g^{\mu \nu}$.

Diagram $(f)$

$$
\begin{aligned}
& \Gamma_{7{ }^{\prime}(f)}^{B M}=-f^{2} g^{2} \operatorname{tr} \int \frac{d^{n} \ell}{(2 \pi)^{n}} \frac{1}{\ell-m} \frac{1}{\ell-m} R \gamma^{\mu} L \frac{1}{\ell-m} \frac{1}{\ell-m} R \gamma^{\nu} L \\
& \Gamma_{Z_{f}(R)}^{R 5}=-f^{2} g^{2} \operatorname{tr} \int \frac{d^{n} \ell}{(2 \pi)^{n}} \frac{1}{\ell-m} \frac{1}{\ell-m} \gamma^{\mu} \frac{2 m \ell}{\left(\ell^{2}-m^{2}\right)^{2}} \gamma^{\nu} L \\
& \lim _{n \rightarrow 4}\left(\Gamma_{\left[\frac{R 5}{7}(f)\right.}-\Gamma_{\left[\frac{B M}{7}(f)\right.}^{B M}\right)=0
\end{aligned}
$$

Diagram $(g)$

$$
\begin{aligned}
& \Gamma_{\left.7 \gamma^{g}\right)}^{B M}=f^{2} g^{2} \operatorname{tr} \int \frac{d^{n} \ell}{(2 \pi)^{n}} \frac{1}{\ell-m} \gamma_{5} \frac{1}{\ell-m} \gamma_{5} \frac{1}{\ell-m} R \gamma^{\mu} L \frac{1}{\ell-m} R \gamma^{\nu} L \\
& \Gamma_{7_{g}^{25}}^{R 5}=-f^{2} g^{2} \operatorname{tr} \int \frac{d^{n} \ell}{(2 \pi)^{n}} \frac{1}{\ell-m} \frac{1}{\ell+m} \frac{1}{\ell-m} \gamma^{\mu} \frac{\ell}{\ell^{2}-m^{2}} \gamma^{\nu} L \\
& \lim _{n \rightarrow 4}\left(\Gamma_{[7(g)}^{R 5}-\Gamma_{[7(g)}^{B M}\right) \\
& =-f^{2} g^{2} \int \frac{d^{n} \ell}{(2 \pi)^{n}} \frac{\operatorname{tr}\left[\ell^{2} \ell \gamma^{\mu} \ell \gamma^{\nu} L+\ell \gamma_{5} \ell \gamma_{5} \ell R \gamma^{\mu} L \ell R \gamma^{\nu} L\right]}{\left(\ell^{2}-m^{2}\right)^{4}} \\
& =\frac{1}{2} f^{2} g^{2} \int \frac{d^{n} \ell}{(2 \pi)^{n}} \frac{\ell_{\Delta}^{2}\left(\ell^{2}+2 \underline{\ell}^{2}\right)}{\left(\ell^{2}-m^{2}\right)^{4}} \operatorname{tr}\left[\gamma^{\mu} \gamma^{\nu}\right]=-\frac{1}{(4 \pi)^{2}} i \frac{7}{3} f^{2} g^{2} g^{\mu \nu}
\end{aligned}
$$

Permuting the two external $\phi_{2}$ and the two external $A$ yields three additional diagrams each of them contributes the same counter-term amplitude as the above. The total counter-term amplitude is therefore equal to $2 \times 2 \times\left(-\frac{1}{(4 \pi)^{2}} i \frac{7}{3} f^{2} g^{2} g^{\mu \nu}\right)=-\frac{1}{(4 \pi)^{2}} i \frac{28}{3} f^{2} g^{2} g^{\mu \nu}$ Diagram $(h)$

$$
\Gamma_{\left.7^{(} h\right)}^{B M}=f^{2} g^{2} \operatorname{tr} \int \frac{d^{n} \ell}{(2 \pi)^{n}} \frac{1}{\ell-m} \gamma_{5} \frac{1}{\ell-m} R \gamma^{\mu} L \frac{1}{\ell-m} \gamma_{5} \frac{1}{\ell-m} R \gamma^{\nu} L
$$

Rightmost- $\gamma_{5}$ amplitude vanishes in the $T_{0}$ order.

$$
\Gamma_{7_{(h)}^{R 5}}^{R 5}=0
$$




$$
\begin{aligned}
& \lim _{n \rightarrow 4}\left(\Gamma_{[7(h)}^{R 5}-\Gamma_{[7(h)}^{B M}\right) \\
& =4 f^{2} g^{2} \operatorname{tr} \int \frac{d^{n} \ell}{(2 \pi)^{n}} \frac{-\ell_{\Delta}^{2} \underline{\ell} \gamma^{\mu} \underline{\ell} \gamma^{\nu} L}{\left(\ell^{2}-m^{2}\right)^{4}}=4 f^{2} g^{2} \frac{4}{n(n+2)} \int \frac{d^{n} \ell}{(2 \pi)^{n}} \frac{(n-4)}{\left(\ell^{2}-m^{2}\right)^{2}} g^{\mu \nu} \\
& =-\frac{1}{(4 \pi)^{2}} \frac{4}{3} i f^{2} g^{2} g^{\mu \nu}
\end{aligned}
$$

By reversing the loop direction, or by exchanging the two external $\phi_{2}$ fields or the two external $A$ fields, we obtain another diagram that also contributes the same counter-term amplitude as the above. The total counter-term amplitude is $2 \times\left(-\frac{1}{(4 \pi)^{2}} \frac{4}{3} i f^{2} g^{2} g^{\mu \nu}\right)=$ $-\frac{1}{(4 \pi)^{2}} \frac{8}{3} i f^{2} g^{2} g^{\mu \nu}$.

\section{$\operatorname{Diagram}(i)$}

$$
\begin{aligned}
& \Gamma_{[]_{i)}^{B M}}^{B M}=-g^{4} \int \frac{d^{n} \ell}{(2 \pi)^{n}} \frac{\operatorname{tr}\left[R \gamma^{\mu} L \ell R \gamma^{\nu} L \ell R \gamma^{\rho} L \ell R \gamma^{\sigma} L\right]}{\left(\ell^{2}-m^{2}\right)^{4}} \\
& \Gamma_{7_{(i)}^{R 5}}^{R 5}=-g^{4} \int \frac{d^{n} \ell}{(2 \pi)^{n}} \frac{\operatorname{tr}\left[\ell \gamma^{\mu} \ell \gamma^{\nu} \ell \gamma^{\rho} \ell \gamma^{\sigma} L\right]}{\left(\ell^{2}-m^{2}\right)^{4}} \\
& \lim _{n \rightarrow 4}\left(\Gamma_{[7(i)}^{R 5}-\Gamma_{[7(i)}^{B M}\right) \\
& =-g^{4} \int \frac{d^{n} \ell}{(2 \pi)^{n}} \frac{\operatorname{tr}\left[\ell \gamma^{\mu} \ell \gamma^{\nu} \ell \gamma^{\rho} \ell \gamma^{\sigma} L-\underline{\ell} \gamma^{\mu} \underline{\ell} \gamma^{\nu} \underline{\ell} \gamma^{\rho} \underline{\ell} \gamma^{\sigma} L\right]}{\left(\ell^{2}-m^{2}\right)^{4}} \\
& =\frac{1}{2} g^{4} \int \frac{d^{n} \ell}{(2 \pi)^{n}} \frac{\ell_{\Delta}^{2} \operatorname{tr}\left[\underline{\ell}^{2}\left(-2 \gamma^{\mu} \gamma^{\nu} \gamma^{\rho} \gamma^{\sigma}+\gamma^{\mu} \gamma^{\sigma} g^{\nu \rho}+g^{\mu \nu} \gamma^{\rho} \gamma^{\sigma}\right)-\ell_{\Delta}^{2} \gamma^{\mu} \gamma^{\nu} \gamma^{\rho} \gamma^{\sigma}\right]}{\left(\ell^{2}-m^{2}\right)^{4}} \\
& =\frac{1}{(4 \pi)^{2}} i g^{4}\left(g^{\mu \nu} g^{\rho \sigma}-\frac{5}{3} g^{\mu \rho} g^{\nu \sigma}+g^{\mu \sigma} g^{\rho \nu}\right)
\end{aligned}
$$

The above amplitude is invariant if we reverse the loop direction or make the interchange $(\mu \leftrightarrow \sigma)$. For the 4-point $A A A A$ 1PI function, there are in total 6 topologically different diagrams that may be obtained from Figure $7(i)$ by permuting the indices $\nu, \rho$, and $\sigma$. The total amplitude for $A A A A$ is equal to

$$
\begin{aligned}
& \frac{1}{(4 \pi)^{2}} i 2 g^{4}\left(g^{\mu \nu} g^{\rho \sigma}-\frac{5}{3} g^{\mu \rho} g^{\nu \sigma}+g^{\mu \sigma} g^{\rho \nu}+(\rho \leftrightarrow \nu)+(\rho \leftrightarrow \sigma)\right) \\
& =\frac{1}{(4 \pi)^{2}} i \frac{2}{3} g^{4}\left(g^{\mu \nu} g^{\rho \sigma}+g^{\mu \rho} g^{\nu \sigma}+g^{\mu \sigma} g^{\rho \nu}\right)
\end{aligned}
$$

\section{Summary}

The amplitudes and finite counter terms due to diagrams in Figure 7 are tabulated in Table X. 


\begin{tabular}{|l|l|l|l|}
\hline Figure & $(4 \pi)^{2} \times\left(\Gamma^{R 5}-\Gamma^{B M}\right)$ & Multiplicity & $(4 \pi)^{2} \times$ Counter Term \\
\hline $7(a)$ & 0 & $3 !$ & 0 \\
\hline $7(b)$ & $4 i f^{4}$ & 4 & $4 f^{4} H^{2}\left(\phi_{2}\right)^{2}$ \\
\hline $7(c)$ & 0 & 2 & 0 \\
\hline $7(d)$ & $\frac{32}{3} i f^{4}$ & $3 !$ & $\frac{8}{3} f^{4}\left(\phi_{2}\right)^{4}$ \\
\hline $7(e)$ & $-i f^{2} g^{2} g^{\mu \nu}$ & 4 & $-f^{2} g^{2} H^{2} A^{2}$ \\
\hline $7(f)$ & 0 & 2 & 0 \\
\hline $7(g)$ & $-i \frac{7}{3} f^{2} g^{2} g^{\mu \nu}$ & $-\frac{7}{3} f^{2} g^{2}\left(\phi_{2}\right)^{2} A^{2}$ \\
\hline $7(h)$ & $-i \frac{4}{3} f^{2} g^{2} g^{\mu \nu}$ & $-\frac{2}{3} f^{2} g^{2}\left(\phi_{2}\right)^{2} A^{2}$ \\
\hline $7(i)+(\rho \leftrightarrow \nu)$ & $i \frac{g^{4}}{3}\left(g^{\mu \nu} g^{\rho \sigma}+g^{\mu \rho} g^{\nu \sigma}\right.$ \\
$+(\rho \leftrightarrow \sigma)$ & 2 & $\frac{1}{12} g^{4}\left(A^{2}\right)^{2}$ \\
\hline
\end{tabular}

TABLE X. Counter terms due to diagrams in Figure 7

\section{Appendix B: The Chiral Non-Abelian Gauge Theory}

\section{Feynman Rules}

The propagators and vertices used in the 1-loop counter-term calculation for the chiral Abelian-Higgs theory defined by (28) are listed below.

a. Propagators:

$$
\begin{aligned}
& S(\psi, \bar{\psi} ; p): \quad \underline{\psi \quad p \quad \bar{\psi}}=\frac{i}{\not p}
\end{aligned}
$$

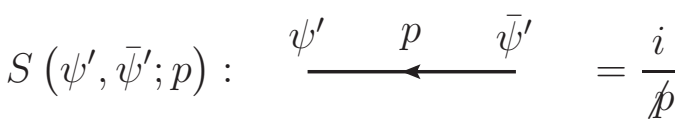

$$
\begin{aligned}
& D\left(A^{a, \mu}, A^{b, \nu} ; k\right): \widetilde{\sim}_{a, \mu}^{\leftarrow} \widetilde{b, \nu}^{\leftarrow}=\frac{-i}{k^{2}}\left(g^{\mu \nu}+(\alpha-1) \frac{k^{\mu} k^{\nu}}{k^{2}}\right) \delta^{a b}
\end{aligned}
$$


b. Vertex Factors:

$$
\begin{aligned}
& \bar{\psi} A^{a, \mu} \psi:-\frac{\sum_{\bar{\psi}} A^{a, \mu}}{\psi}=-i g R \gamma^{\mu} L T_{L}^{a} \\
& \bar{\psi}^{\prime} A^{a, \mu} \psi^{\prime}: \frac{\sum_{\bar{\psi}^{\prime}} A^{a, \mu} \psi^{\prime}}{A^{\prime}}=-i g L \gamma^{\mu} R T_{R}^{a} \\
& A_{\mu}^{a} A_{\nu}^{b} A_{\rho}^{c}: A_{A_{2}, \nu} \sum_{k_{3}}^{a, \mu} \\
& =-g C^{a b c}\left(g^{\mu \nu}\left(k_{1}-k_{2}\right)^{\rho}+g^{\nu \rho}\left(k_{2}-k_{3}\right)^{\mu}+g^{\mu \rho}\left(k_{3}-k_{1}\right)^{\nu}\right)
\end{aligned}
$$

\section{1-Loop Counter Terms}

a. Figure 8: Fermion Self-Energy Diagram

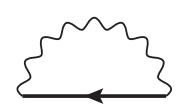

FIG. 8. Fermion Self-Energy Diagram

The fermion field in Figure 8 can be either $\psi$ or $\psi^{\prime}$. If the fermion field is $\psi$, the Feynman integral for Figure 8 without including the non-Abelian group factor $\sum_{e} T_{L}^{e} T_{L}^{e}=C_{L}$ is the same as the one for the diagram of (14) of which the counter term has been demonstrated to be (19) in Sec. IV] If the fermion field is $\psi^{\prime}$, the counter term can be obtained from that for the $\psi$ field by replacing $\gamma_{5}$ with $-\gamma_{5}$ and the group factor $C_{L}$ with $\sum_{e} T_{R}^{e} T_{R}^{e}=C_{R}$. The counter term therefore is equal to

$$
-\frac{1}{(4 \pi)^{2}} \frac{1}{3} g^{2}(1+2 \alpha)\left(\bar{\psi}_{L} i \not \partial \psi_{L} C_{L}+\bar{\psi}_{R}^{\prime} i \not \partial \psi_{R}^{\prime} C_{R}\right)
$$




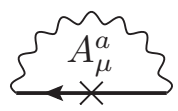

(a)

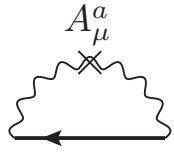

$(b)$

FIG. 9. $\bar{\psi} A \psi$ and $\bar{\psi}^{\prime} A \psi^{\prime}$ Vertex Diagrams

b. Figure 9: $\bar{\psi} A \psi$ and $\bar{\psi}^{\prime} A \psi^{\prime}$ Vertex Diagrams

\section{Diagram $(a)$}

The amplitude for this diagram excluding the non-Abelian group factor is the same as that for Figure 2 $(a)$. If $\psi$ is the fermion field, the non-Abelian group factor is $\sum_{e} T_{L}^{e} T_{L}^{a} T_{L}^{e}=$ $C_{L} T_{L}^{a}+i C^{a b c} T_{L}^{b} T_{L}^{c}$ and

$$
\lim _{n \rightarrow 4}\left(\Gamma_{\psi 9_{(a)}^{R 5}}^{R \Gamma_{\psi}^{B M}(a)}\right)=\frac{1}{(4 \pi)^{2}} \frac{i g^{3}}{6}(7+5 \alpha) \gamma^{\mu} L\left(C_{L} T_{L}^{a}+i C^{a b c} T_{L}^{b} T_{L}^{c}\right)
$$

If $\psi^{\prime}$ is the fermion field, the non-Abelian group factor is $\sum_{e} T_{R}^{e} T_{R}^{a} T_{R}^{e}=C_{R} T_{R}^{a}+i C^{a b c} T_{R}^{b} T_{R}^{c}$ and

$$
\lim _{n \rightarrow 4}\left(\Gamma_{\psi^{\prime}}^{R 5} 9_{(a)}-\Gamma_{\psi^{\prime}}^{B M}(a)\right)=\frac{1}{(4 \pi)^{2}} \frac{i g^{3}}{6}(7+5 \alpha) \gamma^{\mu} R\left(C_{R} T_{R}^{a}+i C^{a b c} T_{R}^{b} T_{R}^{c}\right)
$$

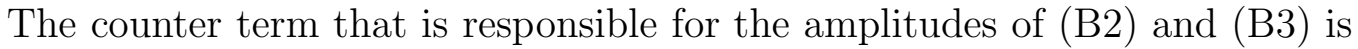

$$
\begin{aligned}
& \frac{1}{6} g^{3}(7+5 \alpha) \bar{\psi}_{L} / A \psi_{L}\left(C_{L} T_{L}^{a}+i C^{a b c} T_{L}^{b} T_{L}^{c}\right) \\
& +\frac{1}{6} g^{3}(7+5 \alpha) \bar{\psi}_{R} / A \psi_{R}\left(C_{R} T_{R}^{a}+i C^{a b c} T_{R}^{b} T_{R}^{c}\right)
\end{aligned}
$$

\section{Diagram $(b)$}

If $\psi$ is the fermion field, we have

$$
\begin{aligned}
\Gamma_{\psi\left[9_{(b)}^{B M}\right.}^{B g^{3}} & =-i \lim _{m \rightarrow 0} \int \frac{d^{n} \ell}{(2 \pi)^{n}} R \gamma^{\tau} L \frac{1}{\ell-m} R \gamma^{\sigma} L C^{b a c} T_{L}^{b} T_{L}^{c} \\
& \times D_{\tau \nu}\left(\ell-k_{1}\right)\left(2 g^{\nu \rho} 2 \ell^{\mu}-g^{\mu \nu} \ell^{\rho}-g^{\mu \rho} \ell^{\nu}\right) D_{\rho \sigma}(\ell)
\end{aligned}
$$


and

$$
\begin{aligned}
& \lim _{n \rightarrow 4}\left(\Gamma_{\psi[\sqrt[9]{(b)}}^{R 5}-\Gamma_{\psi(\underline{9}(b)}^{B M}\right) \\
& =-i g^{3} \lim _{m \rightarrow 0} \int D_{\tau \nu}(\ell)\left(2 g^{\nu \rho} 2 \ell^{\mu}-g^{\mu \nu} \ell^{\rho}-g^{\mu \rho} \ell^{\nu}\right) D_{\rho \sigma}(\ell) \\
& \times \frac{1}{\ell^{2}-m^{2}}\left(\gamma^{\tau} \ell \gamma^{\sigma}-\underline{\gamma}^{\tau} \frac{\ell \gamma^{\sigma}}{}\right) L C^{b a c} T_{L}^{b} T_{L}^{c} \\
& =\frac{1}{(4 \pi)^{2}} \frac{1}{6}(7+5 \alpha) g^{3} \gamma^{\mu} L C^{a b c} T_{L}^{b} T_{L}^{c}
\end{aligned}
$$

If $\psi^{\prime}$ is the fermion field, we have

$$
\begin{aligned}
\Gamma_{\psi^{\prime}}^{B M}(b) & =-i g^{3} \lim _{m \rightarrow 0} \int \frac{d^{n} \ell}{(2 \pi)^{n}} L \gamma^{\tau} R \frac{1}{\ell-m} L \gamma^{\sigma} R C^{b a c} T_{R}^{b} T_{R}^{c} \\
& \times D_{\tau \nu}\left(\ell-k_{1}\right)\left(2 g^{\nu \rho} 2 \ell^{\mu}-g^{\mu \nu} \ell^{\rho}-g^{\mu \rho} \ell^{\nu}\right) D_{\rho \sigma}(\ell)
\end{aligned}
$$

and

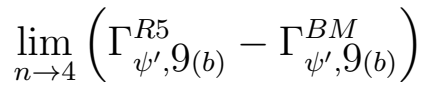

$$
\begin{aligned}
& =-i g^{3} \lim _{m \rightarrow 0} \int D_{\tau \nu}(\ell)\left(2 g^{\nu \rho} 2 \ell^{\mu}-g^{\mu \nu} \ell^{\rho}-g^{\mu \rho} \ell^{\nu}\right) D_{\rho \sigma}(\ell) \\
& \times \frac{1}{\ell^{2}-m^{2}}\left(\gamma^{\tau} \ell \gamma^{\sigma}-\underline{\gamma}^{\tau} \underline{\ell \gamma^{\sigma}}\right) R C^{b a c} T_{R}^{b} T_{R}^{c} \\
& =\frac{1}{(4 \pi)^{2}} \frac{1}{6}(7+5 \alpha) g^{3} \gamma^{\mu} R C^{a b c} T_{R}^{b} T_{R}^{c}
\end{aligned}
$$

The counter term to generate the amplitudes of $(\underline{\mathrm{B} 5})$ and $(\underline{\mathrm{B} 6})$ is

$$
-i \frac{1}{(4 \pi)^{2}} \frac{1}{6} g^{3}(7+5 \alpha)\left(\bar{\psi}_{L} / A \psi_{L} C^{a b c} T_{L}^{b} T_{L}^{c}+\bar{\psi}_{R}^{\prime} / A \psi_{R}^{\prime} C^{a b c} T_{R}^{b} T_{R}^{c}\right)
$$

Summary

The sum of (B4) and (B7) is the total counter term due to the two diagrams in Figure 9 ;

$$
\frac{1}{(4 \pi)^{2}} \frac{1}{6} g^{3}(7+5 \alpha)\left(\bar{\psi}_{L} / A \psi_{L} C_{L} T_{L}^{a}+\bar{\psi}_{R}^{\prime} / A \psi_{R}^{\prime} C_{R} T_{R}^{a}\right)
$$

\section{c. Figure 10: One-Fermion-Loop 2-Point 1PI}

Ignoring the non-Abelian group factor, the diagram in Figure 10 is the same as diagram (c) in Figure 5. For the $\psi$ fermion field, the group factor is $\operatorname{tr}\left(T_{L}^{a} T_{L}^{b}\right)=T_{L} \delta^{a b}$. Consequently,

$$
\lim _{n \rightarrow 4}\left(\Gamma_{\psi[10}^{R 5}-\Gamma_{\psi}^{B M} \underline{10}\right)=\frac{1}{(4 \pi)^{2}} i g^{\mu \nu} \frac{1}{3} g^{2} p^{2} T_{L} \delta^{a b}
$$




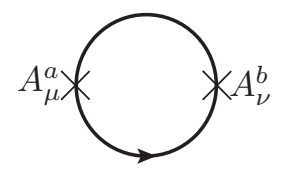

FIG. 10. Diagrams for 2-point 1PI

where $p$ is the external momentum. The group factor for $\psi^{\prime}$ is $\operatorname{tr}\left(T_{R}^{a} T_{R}^{b}\right)=T_{R} \delta^{a b}$ which yields the difference

$$
\lim _{n \rightarrow 4}\left(\Gamma_{\psi^{\prime}}^{R 5}-\Gamma_{\psi^{\prime}[10}^{B M}\right)=\frac{1}{(4 \pi)^{2}} i g^{\mu \nu} \frac{1}{3} g^{2} p^{2} T_{R} \delta^{a b}
$$

The above two amplitudes can be accounted for by the counter term

$$
-\frac{1}{(4 \pi)^{2}} \frac{1}{6} g^{2}\left(T_{L}+T_{R}\right) A_{\mu}^{a} \square A^{a, \mu}
$$

d. Figure 11: One-Fermion-Loop 3-Point 1PI

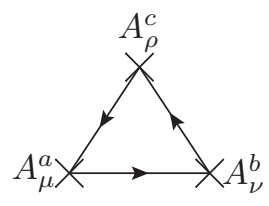

(a)

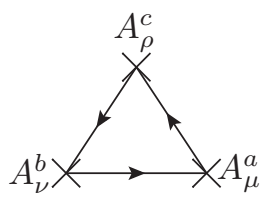

(b)

FIG. 11. Diagrams for 3-point 1PI

We are only interested in terms that have an even count of $\gamma_{5}$. Assume the incoming momenta entering $A_{\mu}^{a}, A_{\nu}^{b}, A_{\rho}^{c}$ are $k_{1}, k_{2}, k_{3}$.

Diagram $(a)$

$$
\begin{aligned}
\Gamma_{\psi \underline{11}_{(a)}^{B M}} & =-g^{3} \lim _{m \rightarrow 0} \operatorname{tr} \int\left(\frac{1}{\ell-m} R \gamma^{\mu} L \frac{1}{\ell-\not k_{1}-m} R \gamma^{\nu} L \frac{1}{\ell+\not k_{3}-m} R \gamma^{\rho} L\right)_{\gamma_{5} \text {-even }} \\
& \times \operatorname{tr}\left(T^{a} T^{b} T^{c}\right)
\end{aligned}
$$




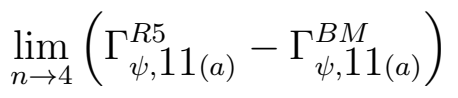

$$
\begin{aligned}
& =-\frac{g^{3}}{2} \lim _{m \rightarrow 0} \int \frac{\operatorname{tr}\left(\not \gamma^{\mu}\left(\ell-\not k_{1}\right) \gamma^{\nu}\left(\ell+\not k_{3}\right) \gamma^{\rho}-\underline{\ell} \gamma^{\mu}\left(\underline{\ell}-\not k_{1}\right) \gamma^{\nu}\left(\underline{\ell}+\not k_{3}\right) \gamma^{\rho}\right)}{\left(\ell^{2}-m^{2}\right)\left(\left(\ell-k_{1}\right)^{2}-m^{2}\right)\left(\left(\ell+k_{3}\right)^{2}-m^{2}\right)} \\
& \times \operatorname{tr}\left(T^{a} T^{b} T^{c}\right) \\
& =-\frac{1}{(4 \pi)^{2}} \frac{2 i}{3} g^{3}\left(\left(k_{2}-k_{3}\right)^{\mu} g^{\nu \rho}+\left(k_{3}-k_{1}\right)^{\nu} g^{\mu \rho}+\left(k_{1}-k_{2}\right)^{\rho} g^{\mu \nu}\right) \operatorname{tr}\left(T_{L}^{a} T_{L}^{b} T_{L}^{c}\right)
\end{aligned}
$$

Similarly,

$$
\begin{aligned}
& \lim _{n \rightarrow 4}\left(\Gamma_{\psi^{\prime}[11}^{R 5}-\Gamma_{\psi^{\prime}[11}^{B M}(a)\right. \\
& =-\frac{1}{(4 \pi)^{2}} \frac{2 i}{3} g^{3}\left(\left(k_{2}-k_{3}\right)^{\mu} g^{\nu \rho}+\left(k_{3}-k_{1}\right)^{\nu} g^{\mu \rho}+\left(k_{1}-k_{2}\right)^{\rho} g^{\mu \nu}\right) \operatorname{tr}\left(T_{R}^{a} T_{R}^{b} T_{R}^{c}\right)
\end{aligned}
$$

Diagram $(b)$

Diagram $(b)$ can be obtained from diagram $(a)$ by the interchange $\left(a, \mu, k_{1}\right) \longleftrightarrow\left(b, \nu, k_{2}\right)$

$$
\begin{aligned}
& \lim _{n \rightarrow 4}\left(\Gamma_{\psi\left[\underline{11}_{b)}^{R 5}-\Gamma_{\psi}^{B M} \underline{11}(b)\right.}\right) \\
& =\frac{1}{(4 \pi)^{2}} \frac{2 i}{3} g^{3}\left(\left(k_{2}-k_{3}\right)^{\mu} g^{\nu \rho}+\left(k_{3}-k_{1}\right)^{\nu} g^{\mu \rho}+\left(k_{1}-k_{2}\right)^{\rho} g^{\mu \nu}\right) \operatorname{tr}\left(T_{L}^{b} T_{L}^{a} T_{L}^{c}\right) \\
& \lim _{n \rightarrow 4}\left(\Gamma_{\psi^{\prime}[11(b)}^{R 5}-\Gamma_{\psi^{\prime}\left[11_{b)}\right.}^{B M}\right) \\
& =\frac{1}{(4 \pi)^{2}} \frac{2 i}{3} g^{3}\left(\left(k_{2}-k_{3}\right)^{\mu} g^{\nu \rho}+\left(k_{3}-k_{1}\right)^{\nu} g^{\mu \rho}+\left(k_{1}-k_{2}\right)^{\rho} g^{\mu \nu}\right) \operatorname{tr}\left(T_{R}^{b} T_{R}^{a} T_{R}^{c}\right)
\end{aligned}
$$

Utilizing $\operatorname{tr}\left(\left[T_{L}^{a}, T_{L}^{b}\right] T_{L}^{c}\right)=i T_{L} C^{a b c}$ and $\operatorname{tr}\left(\left[T_{R}^{a}, T_{R}^{b}\right] T_{R}^{c}\right)=i T_{R} C^{a b c}$, the summation from (B10) to (B13) can be written as

$$
\frac{1}{(4 \pi)^{2}} \frac{2}{3} g^{3}\left(\left(k_{2}-k_{3}\right)^{\mu} g^{\nu \rho}+\left(k_{3}-k_{1}\right)^{\nu} g^{\mu \rho}+\left(k_{1}-k_{2}\right)^{\rho} g^{\mu \nu}\right) C^{a b c}\left(T_{L}+T_{R}\right)
$$

which leads to the counter term

$$
-\frac{1}{(4 \pi)^{2}} \frac{2}{3} g^{3}\left(T_{L}+T_{R}\right) C^{a b c}\left(\partial^{\mu} A_{\nu}^{a}\right) A_{\mu}^{b} A^{c, \nu}
$$

e. Figure 12: One-Fermion-Loop 4-Point 1PI

Diagram $(a)$ 


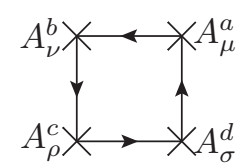

(a)

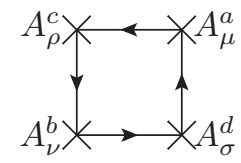

(d)

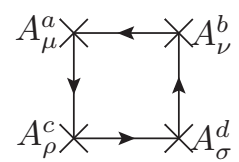

(b)

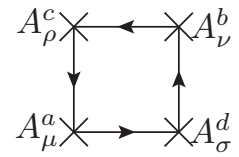

(e)

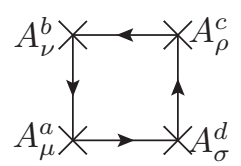

(c)

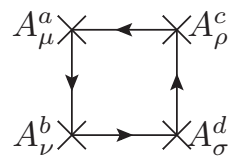

(f)

FIG. 12. Diagrams for 4-point 1PI

If $\psi$ is the fermion field, the amplitude for this diagram is equal to the group factor $\operatorname{tr}\left(T_{L}^{a} T_{L}^{b} T_{L}^{c} T_{L}^{d}\right)=T_{L}^{a b c d}$ times the amplitude for diagram $(i)$ in Figure 7, According to (A2), we then have

$$
\begin{aligned}
& \lim _{n \rightarrow 4}\left(\Gamma_{\psi[12(a)}^{R 5}-\Gamma_{\psi[12(a)}^{B M}\right) \\
& =\frac{1}{(4 \pi)^{2}} i g^{4} T_{L}^{a b c d}\left(g^{\mu \nu} g^{\rho \sigma}-\frac{5}{3} g^{\mu \rho} g^{\nu \sigma}+g^{\mu \sigma} g^{\nu \rho}\right)
\end{aligned}
$$

Likewise, if $\psi^{\prime}$ is the fermion field, $\operatorname{tr}\left(T_{R}^{a} T_{R}^{b} T_{R}^{c} T_{R}^{d}\right)=T_{R}^{a b c d}$ is the group factor and

$$
\begin{aligned}
& \lim _{n \rightarrow 4}\left(\Gamma_{\psi^{\prime} ! 12}^{R 5}(a)-\Gamma_{\psi^{\prime} \underline{12}(a)}^{B M}\right) \\
& =\frac{1}{(4 \pi)^{2}} i g^{4} T_{R}^{a b c d}\left(g^{\mu \nu} g^{\rho \sigma}-\frac{5}{3} g^{\mu \rho} g^{\nu \sigma}+g^{\mu \sigma} g^{\nu \rho}\right)
\end{aligned}
$$

The contribution to the difference from both $\psi$ and $\psi^{\prime}$ fermion loops is the sum of (B15) and (B16), which is equal to

$$
\begin{aligned}
& \lim _{n \rightarrow 4}\left(\Gamma_{\frac{12}{12}_{(a)}}-\Gamma_{\left[\frac{B M}{12}(a)\right.}\right) \\
& =\frac{1}{(4 \pi)^{2}} i g^{4}\left(T_{L}^{a b c d}+T_{R}^{a b c d}\right)\left(g^{\mu \nu} g^{\rho \sigma}-\frac{5}{3} g^{\mu \rho} g^{\nu \sigma}+g^{\mu \sigma} g^{\nu \rho}\right)
\end{aligned}
$$

Diagram $(b)$

The interchange $(a, \mu) \longleftrightarrow(b, \nu)$ on (B17) yields

$$
\begin{aligned}
& \lim _{n \rightarrow 4}\left(\Gamma_{\frac{12}{12}}^{R}(b)-\Gamma_{\frac{12}{12}(b)}^{B d}\right) \\
& =\frac{1}{(4 \pi)^{2}} i g^{4}\left(T_{L}^{b a c d}+T_{R}^{b a c d}\right)\left(g^{\mu \nu} g^{\rho \sigma}+g^{\mu \rho} g^{\nu \sigma}-\frac{5}{3} g^{\mu \sigma} g^{\nu \rho}\right)
\end{aligned}
$$




\section{Diagram $(c)$}

The interchange $(a, \mu) \longleftrightarrow(c, \rho)$ on (B17) yields

$$
\begin{aligned}
& \lim _{n \rightarrow 4}\left(\Gamma_{\frac{12}{12}_{(c)}^{R 5}}-\Gamma_{\left[_{12}(c)\right.}^{B M}\right) \\
& =\frac{1}{(4 \pi)^{2}} i g^{4}\left(T_{L}^{c b a d}+T_{R}^{c b a d}\right)\left(g^{\mu \nu} g^{\rho \sigma}-\frac{5}{3} g^{\mu \rho} g^{\nu \sigma}+g^{\mu \sigma} g^{\nu \rho}\right)
\end{aligned}
$$

Diagram $(d)$

The interchange $(b, \nu) \longleftrightarrow(c, \rho)$ on (B17) yields

$$
\begin{aligned}
& \lim _{n \rightarrow 4}\left(\Gamma_{\left[\frac{12}{12}(d)\right.}^{R 5}-\Gamma_{\frac{12}{12}(d)}^{B M}\right) \\
& =\frac{1}{(4 \pi)^{2}} i g^{4}\left(T_{L}^{a c b d}+T_{R}^{a c b d}\right)\left(-\frac{5}{3} g^{\mu \nu} g^{\rho \sigma}+g^{\mu \rho} g^{\nu \sigma}+g^{\mu \sigma} g^{\nu \rho}\right)
\end{aligned}
$$

Diagram $(e)$

The interchange $(b, \nu) \longleftrightarrow(c, \rho)$ on (B19) yields

$$
\begin{aligned}
& \lim _{n \rightarrow 4}\left(\Gamma_{\frac{12}{12}(e)}-\Gamma_{\frac{12}{12}(e)}^{B M}\right) \\
& =\frac{1}{(4 \pi)^{2}} i g^{4}\left(T_{L}^{b c a d}+T_{R}^{b c a d}\right)\left(-\frac{5}{3} g^{\mu \nu} g^{\rho \sigma}+g^{\mu \rho} g^{\nu \sigma}+g^{\mu \sigma} g^{\nu \rho}\right)
\end{aligned}
$$

Diagram $(f)$

The interchange $(a, \mu) \longleftrightarrow(c, \rho)$ on (B20) yields

$$
\begin{aligned}
& \lim _{n \rightarrow 4}\left(\Gamma_{\frac{R 5}{12}(f)}-\Gamma_{\left[\frac{B M}{12}(f)\right.}\right) \\
& =\frac{1}{(4 \pi)^{2}} i g^{4}\left(T_{L}^{c a b d}+T_{R}^{c a b d}\right)\left(g^{\mu \nu} g^{\rho \sigma}+g^{\mu \rho} g^{\nu \sigma}-\frac{5}{3} g^{\mu \sigma} g^{\nu \rho}\right)
\end{aligned}
$$

The summation from (B17) to (B22) gives

$$
\frac{1}{(4 \pi)^{2}} i g^{4}\left(\begin{array}{c}
g^{\mu \nu} g^{\rho \sigma}\left(\left(T_{L+R}^{a b c d}+T_{L+R}^{b a c d}+T_{L+R}^{c b a d}+T_{L+R}^{c a b d}\right)-\frac{5}{3}\left(T_{L+R}^{a c b d}+T_{L+R}^{b c a d}\right)\right) \\
+g^{\mu \rho} g^{\nu \sigma}\left(\left(T_{L+R}^{b a c d}+T_{L+R}^{c a b d}+T_{L+R}^{a c b d}+T_{L+R}^{b c a d}\right)-\frac{5}{3}\left(T_{L+R}^{a b c d}+T_{L+R}^{c b a d}\right)\right) \\
+g^{\mu \sigma} g^{\nu \rho}\left(\left(T_{L+R}^{a b c d}+T_{L+R}^{c b a d}+T_{L+R}^{a c b d}+T_{L+R}^{b c a d}\right)-\frac{5}{3}\left(T_{L+R}^{b a c d}+T_{L+R}^{c a b d}\right)\right)
\end{array}\right)
$$

where $T_{L+R}^{a b c d}=T_{L}^{a b c d}+T_{R}^{a b c d}$. The above amplitude can be accounted for by the counter term

$$
\begin{aligned}
& \frac{1}{(4 \pi)^{2}} g^{4}\left(\frac{1}{2} T_{L+R}^{a b c d} A^{a, \mu} A_{\mu}^{b} A^{c, \nu} A_{\nu}^{d}-\frac{5}{12} T_{L+R}^{a b c d} A^{a, \mu} A^{b, \nu} A_{\mu}^{c} A_{\nu}^{d}\right) \\
& =\frac{1}{(4 \pi)^{2}} g^{4}\left(\frac{1}{12} T_{L+R}^{a b c d} A^{a, \mu} A_{\mu}^{b} A^{c, \nu} A_{\nu}^{d}+\frac{5}{24}\left(T_{L}+T_{R}\right) C^{e a b} C^{e c d} A_{\mu}^{a} A_{\nu}^{b} A^{c, \mu} A^{d, \nu}\right)
\end{aligned}
$$




\section{f. One-Loop Counter Terms for the Non-Abelian Theory}

The results for the finite counter terms stemming from the difference of amplitudes between the rightmost scheme and the BM scheme calculated for the diagrams in Figure 8, 12 in the chiral non-Abelian gauge theory are summarized in Table XI.

\begin{tabular}{|l|l|l|}
\hline Figure & where & $(4 \pi)^{2} \times$ Counter Term \\
\hline 8 & (B1) & $-\frac{1}{3} g^{2}(1+2 \alpha)\left(\bar{\psi}_{L} i \not \partial \psi_{L} C_{L}+\bar{\psi}_{R}^{\prime} i \not \partial \psi_{R}^{\prime} C_{R}\right)$ \\
\hline 9 & $($ B8 $)$ & $\frac{1}{6} g^{3}(7+5 \alpha)\left(\bar{\psi}_{L} / A \psi_{L} C_{L} T_{L}^{a}+\bar{\psi}_{R}^{\prime} / A \psi_{R} C_{R}^{\prime} T_{R}^{a}\right)$ \\
\hline 10 & $($ B9 $)$ & $-\frac{1}{6} g^{2}\left(T_{L}+T_{R}\right) A_{\mu}^{a} \square A^{a, \mu}$ \\
\hline 11 & $(\overline{B 14})$ & $-\frac{2}{3} g^{3}\left(T_{L}+T_{R}\right) C^{a b c}\left(\partial^{\mu} A_{\nu}^{a}\right) A_{\mu}^{b} A^{c, \nu}$ \\
112 & $(\overline{B 24})$ & $\left(\begin{array}{c}\frac{1}{12} g^{4}\left(T_{L}^{a b c d}+T_{R}^{a b c d}\right) A^{a, \mu} A_{\mu}^{b} A^{c, \nu} A_{\nu}^{d} \\
+\frac{5}{24} g^{4}\left(T_{L}+T_{R}\right) C^{e a b} C^{e c d} A_{\mu}^{a} A_{\nu}^{b} A^{c, \mu} A^{d, \nu}\end{array}\right)$ \\
\hline
\end{tabular}

TABLE XI. Counter terms due to diagrams in Figure 8, 12

[1] G. 't Hooft and M. Veltman, Nucl. Phys. B44, 189 (1972).

[2] P. Breitenlohner and D. Maison, Commun. math. Phys. 52, 11 (1977).

[3] J. C. Ward, Phys. Rev. 78, 182 (1950); Y. Takahashi, Nuovo Cimento, 6, 371 (1957).

[4] C. Becchi, A. Rouet and R. Stora, Phys. Lett. B52, 344 (1974); Comm. Math. Phys. 42, 127 (1975); Ann. of Phys. 98, 287 (1976). I.V. Tyutin, Lebedev Institute preprint 39 (1975).

[5] Guy Bonneau, Nucl. Phys. B177, 523 (1981).

[6] R. Ferrari, A. Le Yaouanc, L. Oliver, and J. C. Raynal, Phys. Rev. D52, 3036 (1995).

[7] R. Ferrari and P. A. Grassi, Phys. Rev. D60, 065010 (1999).

[8] C. P. Martin and D. Sanchez-Ruiz, Nucl. Phys. B572, 387 (2000).

[9] D. Sanchez-Ruiz, Phys. Rev. D68, 025009 (2003).

[10] E. C. Tsai, Gauge Invariant Treatment of $\gamma_{5}$ in the Scheme of 't Hooft and Veltman, arXiv:0905.1550v3 [hep-th]. 